\title{
Combining coordination and hydrogen-bonds to form arene ruthenium metalla-assemblies
}

\author{
Divambal Appavoo, Diego Carnevale, Robert Deschenaux, and Bruno Therrien* \\ Institut de chimie, Université de Neuchâtel, Avenue de Bellevaux 51, CH-2000 Neuchâtel, \\ Switzerland
}

*Corresponding author. Tel: +41 (0)32 718 2499. E-mail address: bruno.therrien@unine.ch

\begin{abstract}
Three ureido-pyrimidone derivatives $\left(\mathrm{N}-\mathrm{L}^{\mathrm{H}}\right)$ were used to generate in combination with four dinuclear arene ruthenium clips, $(p \text {-cymene })_{2} \mathrm{Ru}_{2}(\mathrm{OO} \cap \mathrm{OO}) \mathrm{Cl}_{2}(\mathrm{OO} \cap \mathrm{OO}=$ oxalato, 2,5dioxido-1,4-benzoquinonato, 2,5-dichloro-1,4-benzoquinonato, 5,8-dioxido-1,4naphtoquinonato), twelve cationic arene ruthenium metalla-cycles of the general formula [( $p$ cymene $\left.)_{2} \mathrm{Ru}_{2}(\mathrm{OO} \cap \mathrm{OO})\left(\mathrm{N}-\mathrm{L}^{\mathrm{H}}\right)_{2}\right]_{2}\left(\mathrm{CF}_{3} \mathrm{SO}_{3}\right)_{4}$. The ureido-pyrimidone moieties are able to form strong hydrogen-bonds by self-pairing, thus giving rise to dimeric structures in solution. In addition, to better understand the behavior of the tetranuclear metalla-cycles in solution, the neutral dinuclear arene ruthenium ureido-pyrimidone complexes of the general formula $\{(p$ cymene $\left.) \mathrm{RuCl}_{2}\left(\mathrm{~N}-\mathrm{L}^{\mathrm{H}}\right)\right\}_{2}$ were also prepared and characterized. All metalla-assemblies were studied in solution by NMR spectroscopy, confirming the high stability of the metalla-cycles and the usefulness of hydrogen-bonds in constructing supramolecular assemblies.
\end{abstract}

Keywords: Arene Ruthenium; NMR Spectroscopy; Hydrogen-Bonds; Supramolecular Chemistry; Metalla-Assemblies 


\section{Graphical abstract}

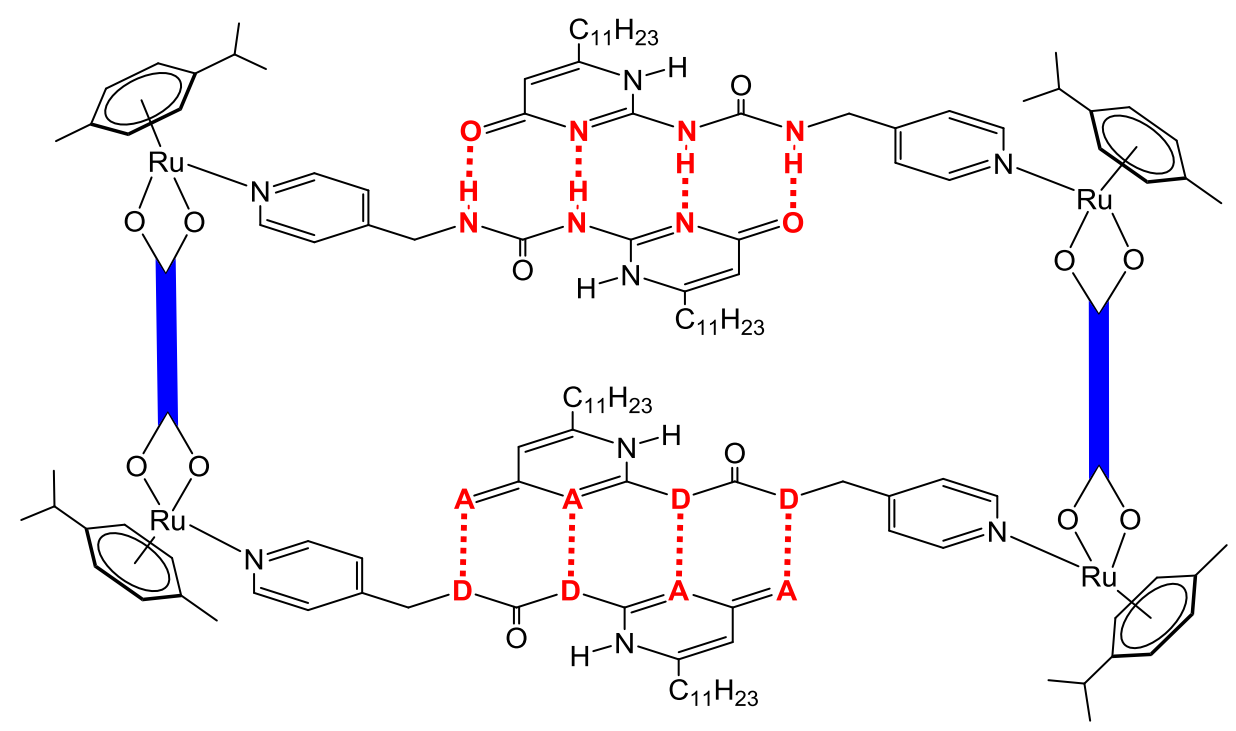

Twelve arene ruthenium metalla-cycles were obtained by combining three ureido-pyrimidone ligands and four dinuclear arene ruthenium complexes. Hydrogen-bonds between the ureidopyrimidone moieties allow the formation of these mixed coordination- and hydrogen-bonded systems. This study confirms the effectiveness of hydrogen-bonds in constructing supramolecular assemblies.

\section{Highlights}

-Twelve arene ruthenium metalla-cycles were synthesized and characterized

-The metalla-cycles are prepared by combining coordination- and hydrogen-bonds

-All metalla-cycles are highly stable in solution 


\section{Introduction}

Hydrogen-bonds have a very important role to play in the structure and function of biological systems. Double strands in DNA are held together by $\pi$-stacking interactions and by hydrogen-bonds between complementary nucleotide Watson-Crick base pairs, which overall give rise to the stable double helical structure of DNA [1]. Similar to the DNA base pairing, the well-defined through-space directionality and hence high selectivity of H-bonds have been used by many researchers to construct supramolecular assemblies [2]. Studies have shown that $\mathrm{H}$-bonds could be made stronger and/or more selective by introducing arrays of donor (D) and acceptor (A) sites. These H-bonded systems are built by the use of parallel arrays of H-bonds and they feature two or more contiguous H-bonds on a heterocyclic scaffold. Units consisting of up to six consecutive H-bonds have been reported [3], but those constituted of triple [4] and quadruple [5] units are the most common derivatives in molecular recognition and self-assembly processes. It was also found that the arrangements of the D and A sites, as well as the solvent in which the complexes are formed, are crucial factors for the strength of the H-bond system [6].

Among the six possible permutations within quadruple $\mathrm{H}$-bonding systems, only three have been studied extensively: ADAD-DADA, AADD-DDAA, ADDA-DAAD. Different scaffolds have been used in designing and building up quadruple H-bonding self-assembled systems, but the most popular one remains the 2-ureido-4-[1H]-pyrimidinone (UPy) scaffold (Figure 1). Upy dimers, with a DDAA H-bond array, show high dimerization constants $\left(\mathrm{K}_{\mathrm{dim}}>10^{7}\right.$ $\mathrm{M}^{-1}$ ) and they can be readily synthesized [7]. The high dimerization constant of UPy arises from limited repulsive secondary interactions and attractive secondary interactions within the motif, as well as an intramolecular $\mathrm{H}$-bond that creates order within the molecule by prearrangement of the DDAA motif. 


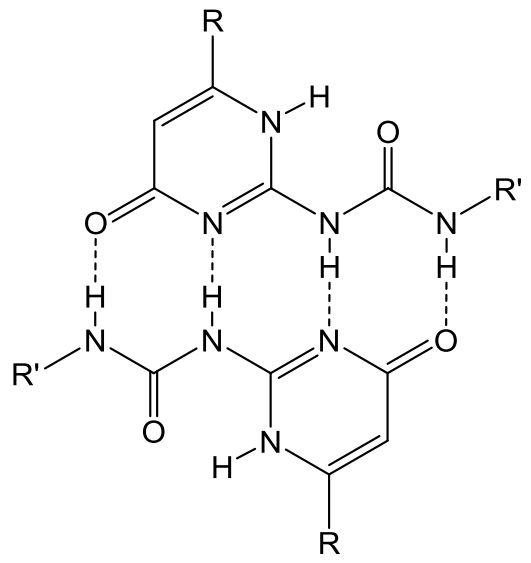

Figure 1. Dimeric structure of a quadruple 2-ureido-4-[1H]-pyrimidinone (UPy) scaffold.

Coordination chemistry is also frequently used to generate supramolecular assemblies [8]. The multivalency and directional ability of metal centers have allowed the construction of two- and three-dimensional assemblies. Among metal centers, the arene ruthenium unit has received considerable attention [9]. The presence of the arene ligand, which occupies three adjacent coordination sites, facilitates the design and formation of discrete metallaassemblies. Combinations of arene ruthenium units with bidentate ligands [10], tridentate ligands [11], tetradentate ligands [12], hexadentate ligands [13], mixtures of bidentate and tridentate [14], bidentate and tetradentate [15], or tridentate and tetradentate ligands [16], have all generated arene ruthenium metalla-cycles and metalla-assemblies. However, we have recently shown that a combination of tetradentate and monodentate ligands can also be successful in making metalla-cycles (Figure 2), if a series of hydrogen-bonds are involved between two monodentate ligands [17]. In this system, the dimeric structure of the two quadruple H-bonding units mimics a bidentate ligand, thus offering new synthetic strategies for the generation of arene ruthenium metalla-assemblies. 


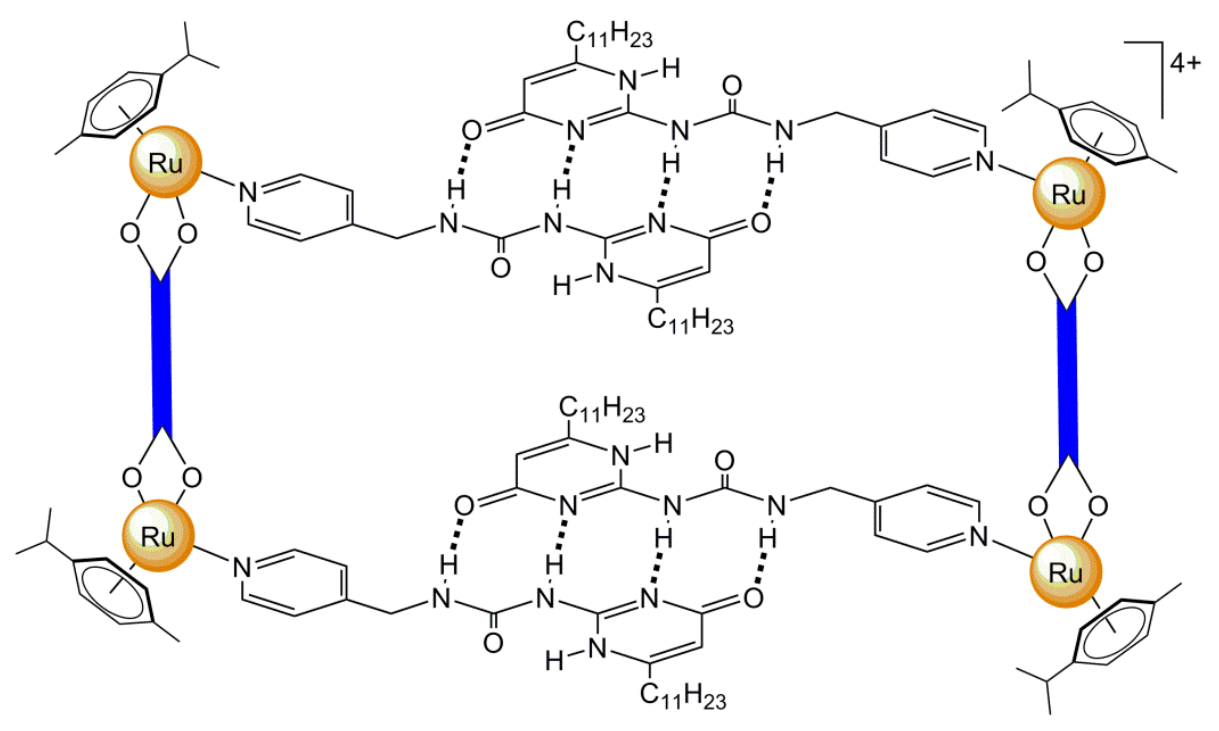

Figure 2. Arene ruthenium metalla-assemblies obtained from a combination of H-bond interactions and tetradentate and monodentate ligands [17].

This strategy has now been extended to two other ureido-pyrimidone derivatives, and three additional tetradentate ligands, thus overall generating twelve tetranuclear arene ruthenium metalla-cycles (including the previously published metalla-cycle $\mathbf{4 b}$ ). All metalla-cycles were studied by spectroscopic and spectrometric methods, and the results were compare to those obtained for the previously published metalla-cycle $\left[(p \text {-cymene })_{2} \mathrm{Ru}_{2}(\mathrm{OO} \cap \mathrm{OO})(\mathrm{N}-\right.$ $\left.\left.\mathrm{L}^{\mathrm{Ha}}\right)_{2}\right]_{2}\left(\mathrm{CF}_{3} \mathrm{SO}_{3}\right)_{4}\left(\mathrm{OO} \cap \mathrm{OO}=2,5\right.$-dioxido-1,4-benzoquinonato; $\mathrm{N}-\mathrm{L}^{\mathrm{Ha}}=1$-(4-oxo-6-undecyl1,4-dihydropyrimidin-2-yl)-3-(pyridine-4-ylmethyl)urea). Overall, this study confirms the stability, versatility and usefulness of hydrogen-bonds in coordination-driven self-assembly. 


\section{Results and discussion}

Following the synthetic strategy developed by Marshall and de Mendoza for 1-(4-oxo-6undecyl-1,4-dihydropyrimidin-2-yl)-3-(pyridin-4-ylmethyl) urea (N-L ${ }^{\mathrm{Ha}}$ ) [18], we have synthesized two additional ureido-pyrimidone derivatives, by replacing at the beginning of the synthetic route the starting material 4-(aminomethyl)pyridine in $\mathrm{N}-\mathrm{L}^{\mathrm{Ha}}$ with respectively 4-(1aminoethyl)pyridine (for N-L ${ }^{\mathrm{Hb}}$ ) and 4-(2-aminoethyl)pyridine (for $\mathrm{N}-\mathrm{L}^{\mathrm{Hc}}$ ) (Figure 3). Like the $\mathrm{N}-\mathrm{L}^{\mathrm{Ha}}$ parent compound, these two derivatives show a dimeric structure in $\mathrm{CDCl}_{3}$ solution, which is confirmed by NMR spectroscopy.



$\mathrm{N}-\mathrm{L}^{\mathrm{Ha}}$



$\mathrm{N}-\mathrm{L}^{\mathrm{Hb}}$

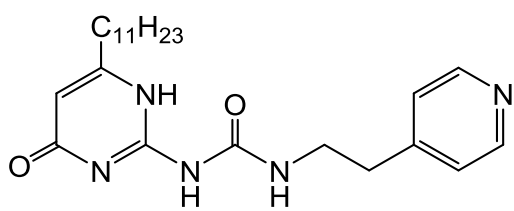

$\mathrm{N}-\mathrm{L}^{\mathrm{Hc}}$

Figure 3. The ureido-pyrimidone derivatives $\left(\mathrm{N}-\mathrm{L}^{\mathrm{H}}\right)$ used in this study.

Exploiting the dimeric nature of the $\mathrm{N}-\mathrm{L}^{\mathrm{H}}$ ligands [17], a series of dinuclear arene ruthenium complexes were prepared by reacting $\left\{(p \text {-cymene }) \mathrm{RuCl}_{2}\right\}_{2}$ with $\mathrm{N}-\mathrm{L}^{\mathrm{H}}$. The dinuclear complexes $\left\{(p \text {-cymene }) \mathrm{RuCl}_{2}\left(\mathrm{~N}-\mathrm{L}^{\mathrm{H}}\right)\right\}_{2}(\mathbf{1}-\mathbf{3})$ were obtained in excellent yields and isolated as red-orange solids (Figure 4). In the electro-spray ionization mass spectra of 1-3 (chloroform/acetonitrile, positive mode), peaks corresponding to $[\mathrm{M}-\mathrm{Cl}]^{+}(\mathrm{M}=\{(p$ cymene) $\left.\mathrm{RuCl}_{2}\left(\mathrm{~N}-\mathrm{L}^{\mathrm{H}}\right)\right\}_{2}$ ) are observed at $\mathrm{m} / \mathrm{z}=1375.1 \mathrm{D}$ for $\mathbf{1}$ and $1403.2 \mathrm{D}$ for $\mathbf{2}$ and $\mathbf{3}$. A difference of $28 \mathrm{D}$ between these peaks is consistent with the proposed dimeric structures of 1-3, as the molecular weight of the N-L $\mathrm{L}^{\mathrm{Ha}}$ and N-L $\mathrm{L}^{\mathrm{Hb}}$ (or N-L ${ }^{\mathrm{Hc}}$ ) derivatives only differs by 14 D (a carbon and two hydrogens). 



2

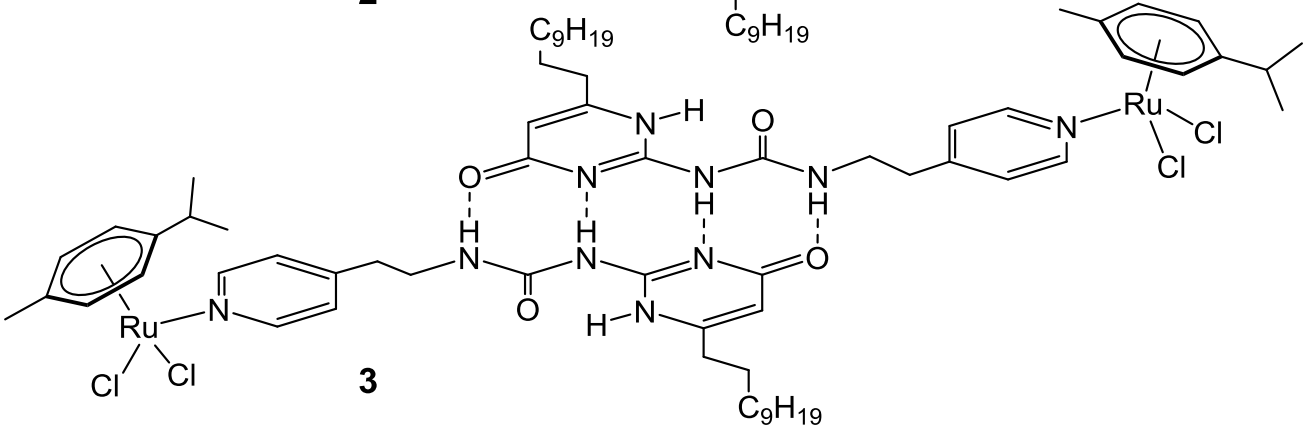

Figure 4. Structures of the dinuclear arene ruthenium complexes $\left\{(p \text {-cymene }) \mathrm{RuCl}_{2}\left(\mathrm{~N}-\mathrm{L}^{\mathrm{H}}\right)\right\}_{2}$ (1-3).

In the ${ }^{1} \mathrm{H}$ NMR spectra of $\mathbf{1}-\mathbf{3}$, the coordination of the dichloro arene ruthenium units to the dimeric $\mathrm{N}-\mathrm{L}^{\mathrm{H}}$ compounds only alters the chemical shift of the pyridyl protons in the orthoposition of the N-L ${ }^{\mathrm{H}}$ ligands. As emphasized in Figure 5, the signal of the ortho-pyridyl protons $\left(\mathrm{H}_{1}\right)$ is shifted downfield by approximately $0.4 \mathrm{ppm}$, while all other signals of the $\mathrm{N}-\mathrm{L}^{\mathrm{H}}$ ligands remain unchanged. Otherwise, the presence of additional signals corresponding to the $p$-cymene ligands is the only difference between the spectra of N-L ${ }^{\mathrm{Ha}}$ and the complex $\left\{\left(p \text {-cymene) } \mathrm{RuCl}_{2}\left(\mathrm{~N}-\mathrm{L}^{\mathrm{Ha}}\right)\right\}_{2}\right.$ (Figure 5). 


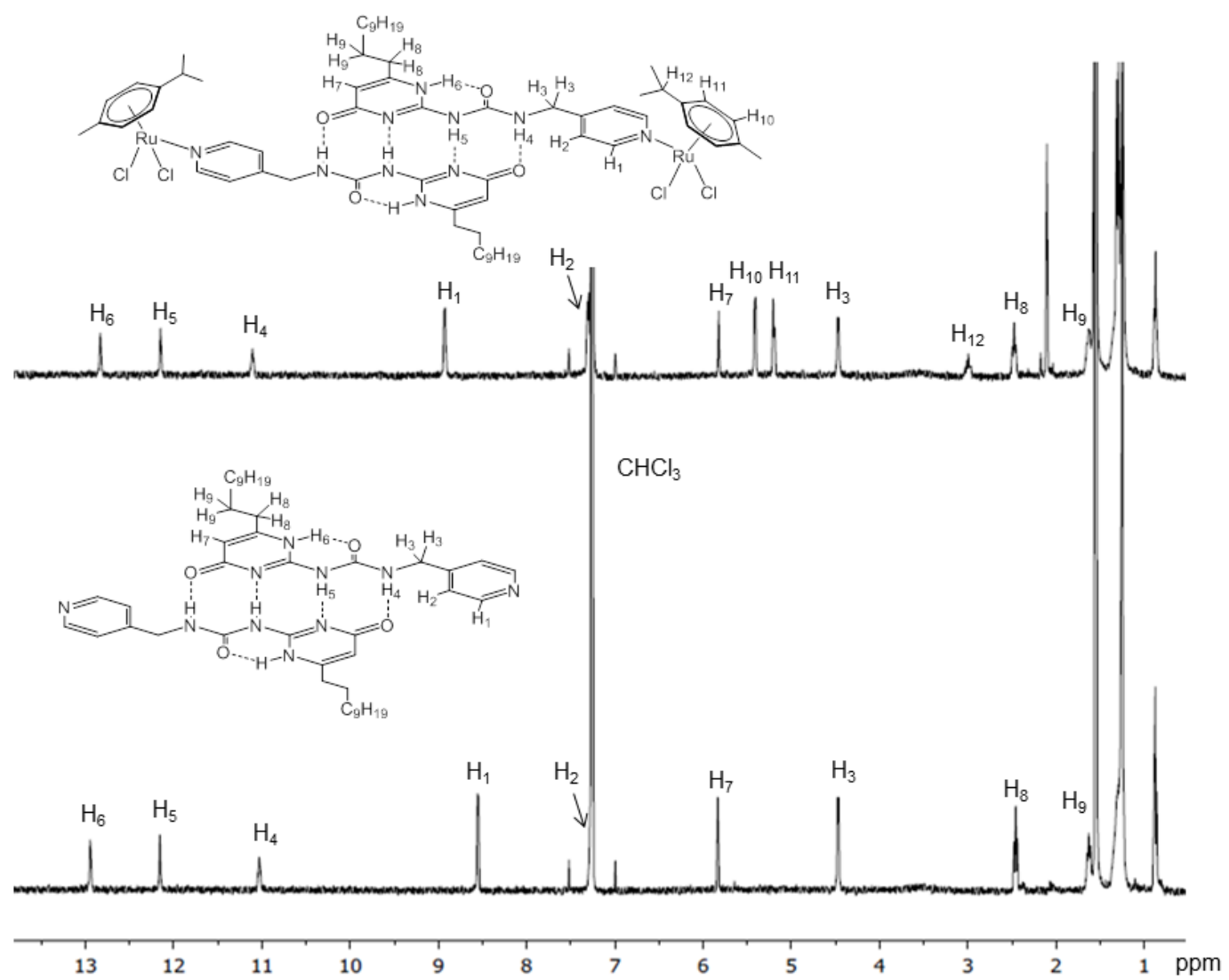

Figure 5. ${ }^{1} \mathrm{H}$ NMR spectra of complex 1 and N-L ${ }^{\mathrm{Ha}}\left(400 \mathrm{MHz}, \mathrm{CDCl}_{3}, 23^{\circ} \mathrm{C}\right)$.

Then, the dinuclear arene ruthenium complexes 1-3 were reacted in dichloromethane with oxalic acid, in the presence of silver triflate, in view to obtain the tetranuclear arene ruthenium metalla-cycles $\left[(p \text {-cymene })_{2} \mathrm{Ru}_{2}(\mathrm{OO} \cap \mathrm{OO})\left(\mathrm{N}-\mathrm{L}^{\mathrm{H}}\right)_{2}\right]_{2}\left(\mathrm{CF}_{3} \mathrm{SO}_{3}\right)_{4}(\mathbf{4 a}-\mathbf{6 a})$. However, under these conditions, the formation of the desired metalla-cycle did not occur: only insoluble materials being recovered after workup. Therefore, we used instead the following strategy to generate the tetranuclear metalla-cycles $\mathbf{4 a - 6 d}$ : Addition of the dinuclear arene ruthenium clips, $(p-$ cymene $)_{2} \mathrm{Ru}_{2}(\mathrm{OO} \cap \mathrm{OO}) \mathrm{Cl}_{2} \quad(\mathrm{OO} \cap \mathrm{OO}=$ oxalato, 2,5-dioxido-1,4-benzoquinonato, 2,5dichloro-1,4-benzoquinonato, 5,8-dioxido-1,4-naphtoquinonato), to $\mathrm{N}-\mathrm{L}^{\mathrm{H}}$ in the presence of silver triflate (see experimental part). The twelve arene ruthenium metalla-cycles are presented in Figure 6. 

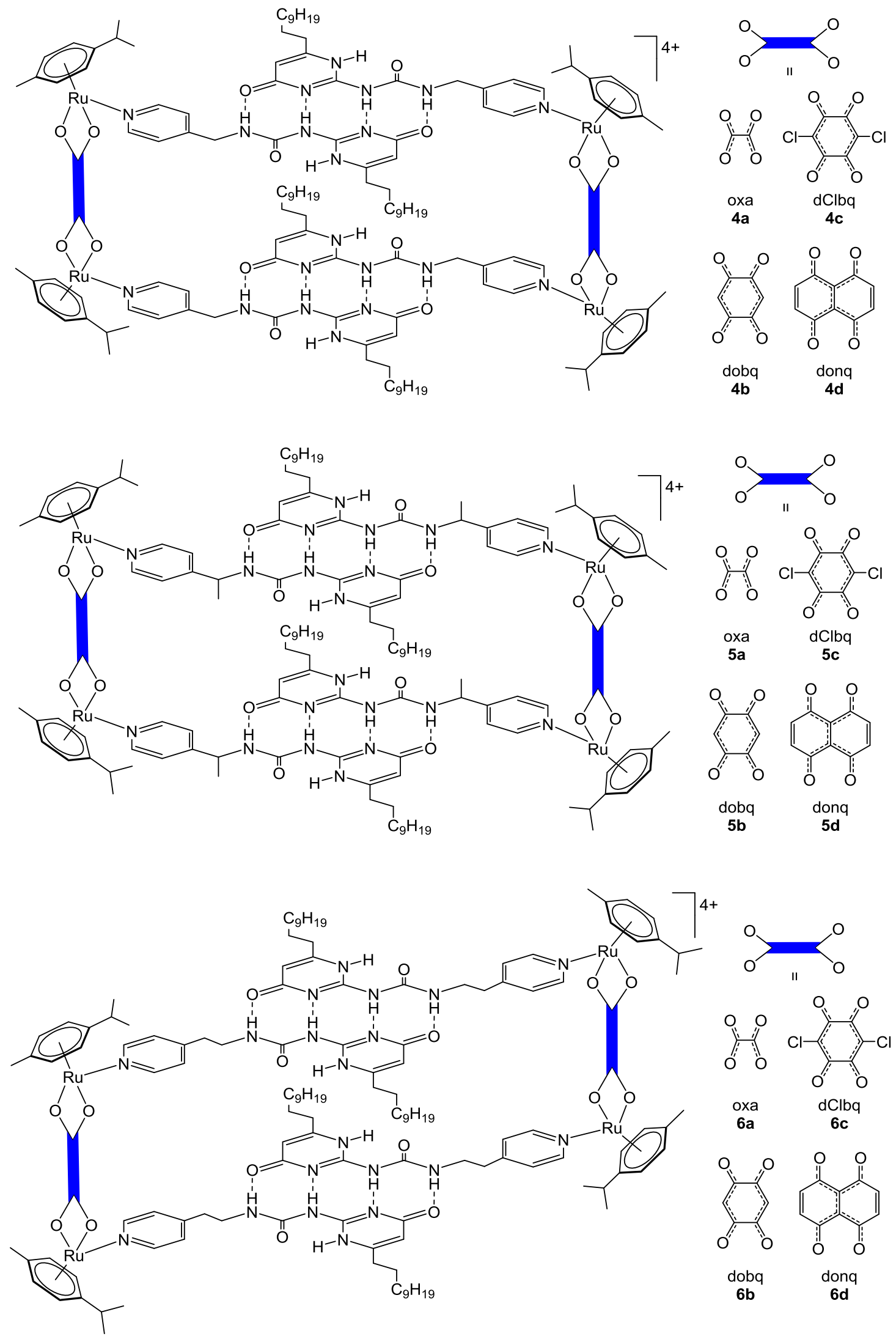

Figure 6. Structures of the twelve tetranuclear arene ruthenium metalla-cycles $[(p-$ cymene $\left.)_{2} \mathrm{Ru}_{2}(\mathrm{OO} \cap \mathrm{OO})\left(\mathrm{N}-\mathrm{L}^{\mathrm{H}}\right)_{2}\right]_{2}\left(\mathrm{CF}_{3} \mathrm{SO}_{3}\right)_{4}(\mathbf{4 a}-\mathbf{6 d})$. 
Upon formation of the tetranuclear complexes $\mathbf{4 a}-\mathbf{6 d}$, alike the dinuclear arene ruthenium complexes 1-3, the ${ }^{1} \mathrm{H}$ NMR signals of the $\mathrm{N}-\mathrm{L}^{\mathrm{H}}$ spacers are almost unaffected. Only the pyridyl protons show a chemical shift variation or a broadening of their signals. Whilst all the ortho-protons of the pyridyl groups are shifted downfield, the broadening of the pyridyl signals is only seldom observed. Such broadening is not surprising considering that the adjacent pyridyl units lose some of their rotational freedom as they coordinate the metal centers and form the metalla-cycles. The same behavior has been previously observed in analogous $\mathrm{OO} \cap \mathrm{OO}-$ bridged arene ruthenium metalla-cycles incorporating bis-pyridyl linkers [19]. The formation of the metalla-cycles $\mathbf{4 a - 6 d}$ is also confirmed by electro-spray ionization mass spectrometry. In all cases, a dicationic peak corresponding to the intact tetracationic metalla-cycle $\left[(p \text {-cymene })_{2} \mathrm{Ru}_{2}(\mathrm{OO} \cap \mathrm{OO})\left(\mathrm{N}-\mathrm{L}^{\mathrm{H}}\right)_{2}\right]_{2}{ }^{4+}$ plus two triflate anions $\left([\mathrm{M}-2 \mathrm{OTf}]^{2+}\right)$ is observed (see experimental part).

Diffusion-ordered NMR spectroscopy (DOSY) was used to estimate the hydrodynamic volumes of the complexes in solution and to confirm the formation of discrete and stable metalla-cycles. All complexes show a single diffusion coefficient, indicative of the presence of single species in solution. The diffusion coefficients $(D)$ of the dinuclear complexes 1-3 are comprised between $4 \times 10^{-10}$ and $5 \times 10^{-10} \mathrm{~m}^{2} \cdot \mathrm{s}^{-1}$ (Figure 7A). Similarly, the diffusion coefficients of the tetranuclear metalla-cycles show values around $4.5 \times 10^{-10} \mathrm{~m}^{2} \cdot \mathrm{s}^{-1}$, and as emphasized in Figure 7B for one representative (4b) of these metalla-cycles, these values are smaller than those observed for the dimeric N-L $\mathrm{L}^{\mathrm{H}}$ systems $\left(D=7.5 \times 10^{-10} \mathrm{~m}^{2} \cdot \mathrm{s}^{-1}\right)$. This difference in the $D$ values is consistent with the difference in sizes between the $\mathrm{N}-\mathrm{L}^{\mathrm{H}}$ dimers and the tetranuclear metalla-cycles, the size being inversely related to $D$. 

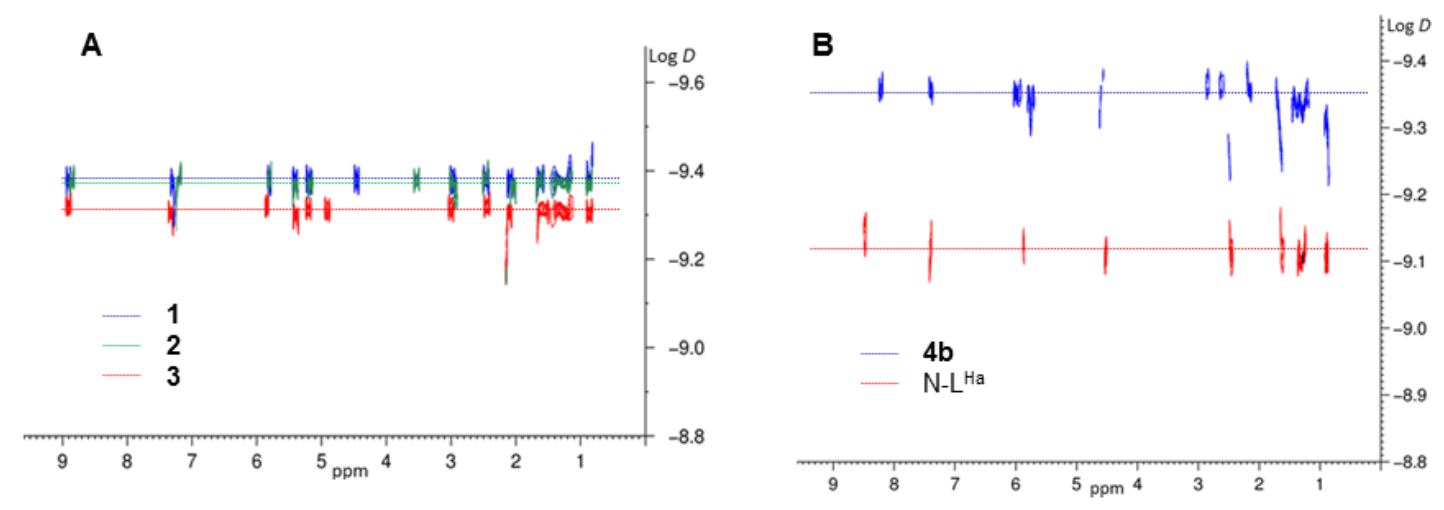

Figure 7. DOSY NMR spectra $\left(\mathrm{CDCl}_{3}, 23^{\circ} \mathrm{C}\right)$ of complexes $\mathbf{1}-\mathbf{3}(\mathbf{A}), \mathrm{N}-\mathrm{L}^{\mathrm{Ha}}$ and $\mathbf{4 b}(\mathbf{B})$.

\section{Conclusion}

Several cationic arene ruthenium metalla-cycles were synthesized and characterized, showing the versatility of hydrogen-bonds in designing supramolecular assemblies. The DDAA hydrogen-bond array present in the ureido-pyrimidone dimers remained stable in solution, even after coordination to the arene ruthenium centers. The synthetic strategy used in this work can be applied to other systems, thus paving the way for the preparation of more elaborate arene ruthenium metalla-assemblies. 


\section{Experimental section}

\subsection{Materials and methods}

All chemicals were purchased from commercial sources and used as received unless specified otherwise. The N-L ${ }^{\mathrm{Ha}}$ ligand [18], and the dinuclear arene ruthenium clips, ( $p$ cymene) ${ }_{2} \mathrm{Ru}_{2}$ (oxalato) $\mathrm{Cl}_{2}$ [15a], ( $p$-cymene) ${ }_{2} \mathrm{Ru}_{2}$ (2,5-dioxido-1,4-benzoquinonato) $\mathrm{Cl}_{2}$ [16a], (p-cymene $)_{2} \mathrm{Ru}_{2}$ (2,5-dichloro-1,4-benzoquinonato) $\mathrm{Cl}_{2}$ [20], and (p-cymene) ${ }_{2} \mathrm{Ru}_{2}$ (5,8-dioxido1,4-naphtoquinonato) $\mathrm{Cl}_{2}$ [21], were prepared according to published methods. The ${ }^{1} \mathrm{H}$ and ${ }^{13} \mathrm{C}\left\{{ }^{1} \mathrm{H}\right\}$ NMR spectra were recorded on a narrow-bore Bruker Avance II $400(9.4 \mathrm{~T})$ spectrometer using the residual protonated solvent as an internal standard. The rf pulses in direct-excitation experiments were set to $30^{\circ}$ flip angles, corresponding to $2.8 \mu$ s for an rffield strength of $27 \mathrm{kHz}$, for both ${ }^{1} \mathrm{H}$ and ${ }^{13} \mathrm{C}$ channels. The recycling delay used in all $1 \mathrm{D}$ spectra was $5 \mathrm{~s}$. DOSY spectra utilized a diffusion delay of $100 \mathrm{~ms}$ and a recycling delay of 1 s. Infrared spectra were recorded as $\mathrm{KBr}$ pellets on a Perkin-Elmer FTIR $1720 \mathrm{X}$ spectrometer. Electrospray ionization mass spectra of the complexes were obtained in positive ion mode on a Bruker FTMS 4.7T BioAPEX II mass spectrometer. UV-visible absorption spectra were recorded in $\mathrm{CH}_{2} \mathrm{Cl}_{2}$ on a Perkin Elmer UV-visible spectrophotometer at $10^{-5} \mathrm{M}$ concentrations.

\subsection{Synthesis of $N-L^{\mathrm{Hb}}$}

4-(1-Aminoethyl)pyridine $\quad(0.11 \quad \mathrm{~g}, \quad 0.91 \quad \mathrm{mmol}) \quad$ and $\quad N$-(6-oxo-4-undecyl-1,6dihydropyrimidin-2-yl)-1H-imidazole-1-carboxamide $(0.35 \mathrm{~g}, 1.00 \mathrm{mmol})$ were placed in a sealed tube containing dry DMF $(5 \mathrm{~mL})$ and triethylamine $(1.5 \mathrm{~mL})$. To the solution, triethylamine $(7.5 \mathrm{~mL})$ was added and the mixture was warmed to $70^{\circ} \mathrm{C}$ overnight. After cooling to $\mathrm{rt}$, the solvent was removed under vacuum and the residue was triturated in methanol. The mixture was filtered and the residue washed with methanol, chloroform and ethyl acetate. $\mathrm{N}-\mathrm{L}^{\mathrm{Hb}}$ was obtained as a white solid with a yield of $50 \%(0.19 \mathrm{~g})$.

${ }^{1} \mathrm{H}$ NMR (400 MHz, $\left.\mathrm{CDCl}_{3}, \mathrm{ppm}\right): \delta=12.91(\mathrm{~s}, 2 \mathrm{H}), 12.10(\mathrm{~s}, 2 \mathrm{H}), 10.81\left(\mathrm{~d},{ }^{3} J_{\mathrm{H}-\mathrm{H}}=7.2 \mathrm{~Hz}\right.$, $2 \mathrm{H}), 8.52\left(\mathrm{~d},{ }^{3} J_{\mathrm{H}-\mathrm{H}}=6.0 \mathrm{~Hz}, 4 \mathrm{H}\right), 7.31(\mathrm{~m}, 4 \mathrm{H}), 5.87(\mathrm{~s}, 2 \mathrm{H}), 4.92$ (quint, $\left.{ }^{3} J_{\mathrm{H}-\mathrm{H}}=7.0 \mathrm{~Hz}, 2 \mathrm{H}\right)$, $2.43\left(\mathrm{t},{ }^{3} J_{\mathrm{H}-\mathrm{H}}=7.7 \mathrm{~Hz}, 4 \mathrm{H}\right), 1.56-1.63(\mathrm{~m}, 10 \mathrm{H}), 1.25-1.28(\mathrm{~m}, 32 \mathrm{H}), 0.86\left(\mathrm{t},{ }^{3} J_{\mathrm{H}-\mathrm{H}}=6.4 \mathrm{~Hz}\right.$, $6 \mathrm{H}) .{ }^{13} \mathrm{C}\left\{{ }^{1} \mathrm{H}\right\} \mathrm{NMR}\left(100 \mathrm{MHz}, \mathrm{CDCl}_{3}, \mathrm{ppm}\right): \delta=173.08 ; 156.10 ; 154.60 ; 153.40 ; 152.60$; $150.09 ; 121.16 ; 106.12 ; 49.42 ; 32.71 ; 31.91 ; 29.58 ; 29.56 ; 29.42 ; 29.33 ; 29.15 ; 28.86 ; 26.96$; 22.70; 21.98; 14.14. IR (KBr) $\left(\mathrm{cm}^{-1}\right)=1695(\mathrm{~s}, \mathrm{NH}), 1662(\mathrm{~s}, \mathrm{NH}), 1593(\mathrm{~s}, \mathrm{NH}) ; 1265$ (s, $\mathrm{CN})$. 


\subsection{Synthesis of $\mathrm{N}-\mathrm{L}^{\mathrm{Hc}}$}

4-(2-Aminoethyl)pyridine $\quad(0.11 \quad \mathrm{~g}, \quad 0.91 \quad \mathrm{mmol}) \quad$ and $\quad N$-(6-oxo-4-undecyl-1,6dihydropyrimidin-2-yl)-1H-imidazole-1-carboxamide $(0.35 \mathrm{~g}, 1.00 \mathrm{mmol})$ were reacted following the procedure for $\mathrm{N}-\mathrm{L}^{\mathrm{Hb}}$ in $\mathrm{DMF}(5 \mathrm{~mL})$ and triethylamine $(1.5 \mathrm{~mL})$ at $70^{\circ} \mathrm{C}$ overnight. N-L ${ }^{\mathrm{Hc}}$ was obtained as a white solid with a yield of $72 \%(0.27 \mathrm{~g})$.

${ }^{1} \mathrm{H}$ NMR (400 MHz, $\left.\mathrm{CDCl}_{3}, \mathrm{ppm}\right): \delta=13.02(\mathrm{~s}, 2 \mathrm{H}), 11.91(\mathrm{~s}, 2 \mathrm{H}), 10.42\left(\mathrm{t},{ }^{3} J_{\mathrm{H}-\mathrm{H}}=5.4 \mathrm{~Hz}\right.$, $2 \mathrm{H}), 8.49\left(\mathrm{~d},{ }^{3} J_{\mathrm{H}-\mathrm{H}}=6.1 \mathrm{~Hz}, 4 \mathrm{H}\right), 7.18\left(\mathrm{~d},{ }^{3} J_{\mathrm{H}-\mathrm{H}}=6.1 \mathrm{~Hz}, 4 \mathrm{H}\right), 5.81(\mathrm{~s}, 2 \mathrm{H}), 3.54\left(\mathrm{dt},{ }^{3} J_{\mathrm{H}-\mathrm{H}}=\right.$ $\left.8.1 \mathrm{~Hz},{ }^{4} J_{\mathrm{H}-\mathrm{H}}=6.1 \mathrm{~Hz}, 4 \mathrm{H}\right), 2.94\left(\mathrm{t},{ }^{3} J_{\mathrm{H}-\mathrm{H}}=7.5 \mathrm{~Hz}, 4 \mathrm{H}\right), 2.47\left(\mathrm{t},{ }^{3} J_{\mathrm{H}-\mathrm{H}}=7.7 \mathrm{~Hz}, 4 \mathrm{H}\right), 1.64$ (quint, $\left.{ }^{3} J_{\mathrm{H}-\mathrm{H}}=7.6 \mathrm{~Hz}, 4 \mathrm{H}\right), 1.26-1.38(\mathrm{~m}, 32 \mathrm{H}), 0.87\left(\mathrm{t},{ }^{3} J_{\mathrm{H}-\mathrm{H}}=6.5 \mathrm{~Hz}, 6 \mathrm{H}\right) .{ }^{13} \mathrm{C}\left\{{ }^{1} \mathrm{H}\right\} \mathrm{NMR}$ $\left(100 \mathrm{MHz}, \mathrm{CDCl}_{3}, \mathrm{ppm}\right): \delta=173.12 ; 156.66 ; 154.59 ; 152.66 ; 149.82 ; 148.09 ; 124.23$; $105.91 ; 40.21 ; 34.97 ; 32.73 ; 31.91 ; 29.59 ; 29.58 ; 29.45 ; 29.33 ; 29.18 ; 28.87 ; 26.99 ; 22.77$; 14.13. IR $(\mathrm{KBr})\left(\mathrm{cm}^{-1}\right)=1702(\mathrm{~s}, \mathrm{NH}), 1654(\mathrm{~s}, \mathrm{NH}), 1579(\mathrm{~s}, \mathrm{NH}) ; 1256(\mathrm{~s}, \mathrm{CN})$.

\subsection{Synthesis of 1-3}

A mixture of $\left\{\left(\eta^{6} \text {-arene }\right) \mathrm{Ru}\left(\mu_{2}-\mathrm{Cl}\right) \mathrm{Cl}\right\}_{2}(0.03 \mathrm{~g}, 0.06 \mathrm{mmol})$ and $\mathrm{N}-\mathrm{L}^{\mathrm{H}}\left(\mathrm{N}-\mathrm{L}^{\mathrm{Ha}}, 0.04 \mathrm{~g}, 0.06\right.$ mmol; N-L ${ }^{\mathrm{Hb}}, 0.05 \mathrm{~g}, 0.06 \mathrm{mmol}$; N-L $\left.\mathrm{L}^{\mathrm{Hc}}, 0.05 \mathrm{~g}, 0.06 \mathrm{mmol}\right)$ is stirred in $\mathrm{CHCl}_{3}(2 \mathrm{~mL})$ at room temperature for 16 hours. Diethyl ether was added to precipitate the product, which was isolated by filtration and dried under vacuum.

1: Yield = 98\% (0.17 g). ${ }^{1} \mathrm{H}$ NMR (400 MHz, $\left.\mathrm{CDCl}_{3}, \mathrm{ppm}\right): \delta=12.83(\mathrm{~s}, 2 \mathrm{H}), 12.15(\mathrm{~s}, 2 \mathrm{H})$, $11.10(\mathrm{~s}, 2 \mathrm{H}), 8.92\left(\mathrm{~d},{ }^{3} J_{\mathrm{H}-\mathrm{H}}=6.0 \mathrm{~Hz}, 4 \mathrm{H}\right), 7.30-7.33\left(\mathrm{~d},{ }^{3} J_{\mathrm{H}-\mathrm{H}}=5.8 \mathrm{~Hz}, 4 \mathrm{H}\right), 5.82(\mathrm{~s}, 2 \mathrm{H})$, $5.40\left(\mathrm{~d},{ }^{3} J_{\mathrm{H}-\mathrm{H}}=5.8 \mathrm{~Hz}, 4 \mathrm{H}\right), 5.19\left(\mathrm{~d},{ }^{3} J_{\mathrm{H}-\mathrm{H}}=5.8 \mathrm{~Hz}, 4 \mathrm{H}\right), 4.46\left(\mathrm{~d},{ }^{3} J_{\mathrm{H}-\mathrm{H}}=5.7 \mathrm{~Hz}, 4 \mathrm{H}\right), 2.99$ (sept, $\left.{ }^{3} J_{\mathrm{H}-\mathrm{H}}=6.9 \mathrm{~Hz}, 2 \mathrm{H}\right), 2.47\left(\mathrm{t},{ }^{3} J_{\mathrm{H}-\mathrm{H}}=7.8 \mathrm{~Hz}, 4 \mathrm{H}\right), 2.10(\mathrm{~s}, 6 \mathrm{H}), 1.63$ (quint, ${ }^{3} J_{\mathrm{H}-\mathrm{H}}=7.5$ $\mathrm{Hz}, 4 \mathrm{H}), 1.23-1.36(\mathrm{~m}, 44 \mathrm{H}), 0.87\left(\mathrm{t},{ }^{3} J_{\mathrm{H}-\mathrm{H}}=6.8 \mathrm{~Hz}, 6 \mathrm{H}\right) .{ }^{13} \mathrm{C}\left\{{ }^{1} \mathrm{H}\right\} \mathrm{NMR}\left(100 \mathrm{MHz}, \mathrm{CDCl}_{3}\right.$, ppm): $\delta=194.92 ; 156.76 ; 154.60 ; 152.99 ; 123.17 ; 106.01 ; 103.60 ; 82.63 ; 82.35 ; 31.84$; $30.61 ; 29.53 ; 29.41 ; 29.27 ; 29.10 ; 28.83 ; 26.88 ; 22.64 ; 22.27 ; 18.29 ; 14.09 ; 0.97$. IR (KBr) $\left(\mathrm{cm}^{-1}\right)=3421(\mathrm{br}, \mathrm{NH}) ; 1697(\mathrm{~s}, \mathrm{NH}) ; 1660(\mathrm{~s}, \mathrm{NH}) ; 1587(\mathrm{~s}, \mathrm{NH}) ; 1257$ (s, CN). MS $(\mathrm{ESI}(+))=1375.1[\mathrm{M}-\mathrm{Cl}]^{+}$. UV-Vis $\left(\mathrm{CH}_{2} \mathrm{Cl}_{2}, 1.0 \times 10^{-5} \mathrm{M}\right): \lambda_{\max }=408 \mathrm{~nm}\left(\varepsilon=0.15 \times 10^{5}\right.$ $\left.\mathrm{M}^{-1} \mathrm{~cm}^{-1}\right)$. 
2: Yield = 93\% (0.16 g). ${ }^{1} \mathrm{H} \mathrm{NMR}\left(400 \mathrm{MHz}, \mathrm{CDCl}_{3}, \mathrm{ppm}\right): \delta=12.81(\mathrm{~s}, 2 \mathrm{H}), 12.09$ (s, 2H), $10.90\left(\mathrm{~d},{ }^{3} J_{\mathrm{H}-\mathrm{H}}=6.9 \mathrm{~Hz}, 2 \mathrm{H}\right), 8.90\left(\mathrm{~d},{ }^{3} J_{\mathrm{H}-\mathrm{H}}=5.7 \mathrm{~Hz}, 4 \mathrm{H}\right), 7.32\left(\mathrm{~d},{ }^{3} J_{\mathrm{H}-\mathrm{H}}=4.6 \mathrm{~Hz}, 4 \mathrm{H}\right), 5.84$ $(\mathrm{s}, 2 \mathrm{H}), 5.39\left(\mathrm{q},{ }^{3} J_{\mathrm{H}-\mathrm{H}}=5.4 \mathrm{~Hz}, 4 \mathrm{H}\right), 5.20\left(\mathrm{q},{ }^{3} J_{\mathrm{H}-\mathrm{H}}=4.7 \mathrm{~Hz}, 4 \mathrm{H}\right), 4.91$ (quint, ${ }^{3} J_{\mathrm{H}-\mathrm{H}}=7.1 \mathrm{~Hz}$, $2 \mathrm{H}), 3.00\left(\mathrm{sept},{ }^{3} J_{\mathrm{H}-\mathrm{H}}=7.1 \mathrm{~Hz}, 2 \mathrm{H}\right), 2.44\left(\mathrm{t},{ }^{3} J_{\mathrm{H}-\mathrm{H}}=7.8 \mathrm{~Hz}, 4 \mathrm{H}\right), 2.10(\mathrm{~s}, 6 \mathrm{H}), 1.63$ (quint, ${ }^{3} J_{\mathrm{H}-}$ $\mathrm{H}=7.5 \mathrm{~Hz}, 4 \mathrm{H}), 1.23-1.30(\mathrm{~m}, 44 \mathrm{H}), 0.86\left(\mathrm{t},{ }^{3} \mathrm{~J}_{\mathrm{H}-\mathrm{H}}=6.6 \mathrm{~Hz}, 6 \mathrm{H}\right) .{ }^{13} \mathrm{C}\left\{{ }^{1} \mathrm{H}\right\} \mathrm{NMR}(100 \mathrm{MHz}$, $\left.\mathrm{CDCl}_{3}, \mathrm{ppm}\right): \delta=173.01 ; 165.76 ; 155.92 ; 154.72 ; 154.34 ; 152.76 ; 122.18 ; 115.94 ; 106.04$; $103.77 ; 96.75 ; 82.44 ; 82.33 ; 49.08 ; 32.63 ; 31.85 ; 30.58 ; 29.53 ; 29.39 ; 29.27 ; 29.10 ; 28.82$; $26.83 ; 22.64 ; 22.23 ; 21.51 ; 18.21 ; 14.09$. IR $(\mathrm{KBr})\left(\mathrm{cm}^{-1}\right)=3421$ (br, NH); 1698 (s, NH); $1658(\mathrm{~s}, \mathrm{NH}) ; 1585$ (s, NH); 1255 (s, CN). MS (ESI(+)) = $1403.2[\mathrm{M}-\mathrm{Cl}]^{+}$. UV-Vis $\left(\mathrm{CH}_{2} \mathrm{Cl}_{2}\right.$, $\left.1.0 \times 10^{-5} \mathrm{M}\right): \lambda_{\max }=409 \mathrm{~nm}\left(\varepsilon=0.18 \times 10^{5} \mathrm{M}^{-1} \mathrm{~cm}^{-1}\right)$.

3: Yield $=96 \%(0.17 \mathrm{~g}) .{ }^{1} \mathrm{H}$ NMR (400 MHz, $\left.\mathrm{CDCl}_{3}, \mathrm{ppm}\right): \delta=12.91(\mathrm{~s}, 2 \mathrm{H}), 11.86(\mathrm{~s}, 2 \mathrm{H})$, $10.41(\mathrm{~s}, 2 \mathrm{H}), 8.85\left(\mathrm{~d},{ }^{3} J_{\mathrm{H}-\mathrm{H}}=5.7 \mathrm{~Hz}, 4 \mathrm{H}\right), 7.20\left(\mathrm{~d},{ }^{3} J_{\mathrm{H}-\mathrm{H}}=5.7 \mathrm{~Hz}, 4 \mathrm{H}\right), 5.79(\mathrm{~s}, 2 \mathrm{H}), 5.40(\mathrm{~d}$, $\left.{ }^{3} J_{\mathrm{H}-\mathrm{H}}=5.7 \mathrm{~Hz}, 4 \mathrm{H}\right), 5.18\left(\mathrm{~d},{ }^{3} J_{\mathrm{H}-\mathrm{H}}=5.7 \mathrm{~Hz}, 4 \mathrm{H}\right), 3.53\left(\mathrm{q},{ }^{3} J_{\mathrm{H}-\mathrm{H}}=6.5 \mathrm{~Hz}, 4 \mathrm{H}\right), 2.92-2.99(\mathrm{~m}$, $6 \mathrm{H}), 2.47\left(\mathrm{t},{ }^{3} J_{\mathrm{H}-\mathrm{H}}=7.7 \mathrm{~Hz}, 4 \mathrm{H}\right), 2.04(\mathrm{~s}, 6 \mathrm{H}), 1.66$ (quint, $\left.{ }^{3} J_{\mathrm{H}-\mathrm{H}}=7.6 \mathrm{~Hz}, 4 \mathrm{H}\right), 1.24-1.37(\mathrm{~m}$, $44 \mathrm{H}), 0.86\left(\mathrm{t},{ }^{3} J_{\mathrm{H}-\mathrm{H}}=6.6 \mathrm{~Hz}, 6 \mathrm{H}\right) .{ }^{13} \mathrm{C}\left\{{ }^{1} \mathrm{H}\right\} \mathrm{NMR}\left(100 \mathrm{MHz}, \mathrm{CDCl}_{3}, \mathrm{ppm}\right): \delta=173.12$; $156.51 ; 154.36 ; 152.79 ; 150.66 ; 125.13 ; 105.80 ; 103.39 ; 96.96 ; 82.79 ; 82.14 ; 39.57 ; 34.27$; $32.68 ; 32.85 ; 30.58 ; 29.53 ; 29.41 ; 29.27 ; 29.13 ; 28.86 ; 26.88 ; 22.64 ; 22.26 ; 18.17 ; 14.10$. IR $(\mathrm{KBr})\left(\mathrm{cm}^{-1}\right)=3425(\mathrm{br}, \mathrm{NH}) ; 1698(\mathrm{~s}, \mathrm{NH}) ; 1659(\mathrm{~s}, \mathrm{NH}) ; 1586(\mathrm{~s}, \mathrm{NH}) ; 1255(\mathrm{~s}, \mathrm{CN}) . \mathrm{MS}$ $(\operatorname{ESI}(+))=1403.2[\mathrm{M}-\mathrm{Cl}]^{+}$. UV-Vis $\left(\mathrm{CH}_{2} \mathrm{Cl}_{2}, 1.0 \times 10^{-5} \mathrm{M}\right): \lambda_{\max }=402 \mathrm{~nm}\left(\varepsilon=0.18 \times 10^{5}\right.$ $\left.\mathrm{M}^{-1} \mathrm{~cm}^{-1}\right)$.

\subsection{Synthesis of $[4 a-6 d]\left(\mathrm{CF}_{3} \mathrm{SO}_{3}\right)_{4}$}

A mixture of 1 equiv. of (p-cymene $)_{2} \mathrm{Ru}_{2}(\mathrm{OO} \cap \mathrm{OO}) \mathrm{Cl}_{2}(0.04 \mathrm{~g}, 0.06 \mathrm{mmol})$ and 3 equiv. of $\mathrm{AgCF}_{3} \mathrm{SO}_{3}(0.04 \mathrm{~g}, 0.18 \mathrm{mmol})$ in $\mathrm{CH}_{2} \mathrm{Cl}_{2}(4 \mathrm{~mL})$ was stirred at room temperature for $2 \mathrm{~h}$ and filtered to remove $\mathrm{AgCl}$. Then $\mathrm{N}-\mathrm{L}^{\mathrm{H}}(0.04 \mathrm{~g}, 0.06 \mathrm{mmol})$ was added to the filtrate and the solution was stirred at room temperature overnight. The volume of the solvent was halved and diethyl ether was added to precipitate $\left[(p \text {-cymene })_{2} \mathrm{Ru}_{2}(\mathrm{OO} \cap \mathrm{OO})\left(\mathrm{N}-\mathrm{L}^{\mathrm{H}}\right)_{2}\right]_{2}\left(\mathrm{CF}_{3} \mathrm{SO}_{3}\right)_{4}$, which was collected as dark solids by filtration.

4a: Yield $=38 \%(0.08 \mathrm{~g}) .{ }^{1} \mathrm{H}$ NMR (400 MHz, $\left.\mathrm{CD}_{3} \mathrm{CN}, \mathrm{ppm}\right): \delta=12.81(\mathrm{~s}, 4 \mathrm{H}), 12.06(\mathrm{~s}$, $4 \mathrm{H}), 10.98(\mathrm{~s}, 4 \mathrm{H}), 8.29-8.38(\mathrm{~m}, 8 \mathrm{H}), 7.85-7.88(\mathrm{~m}, 8 \mathrm{H}), 5.66-5.81(\mathrm{~m}, 12 \mathrm{H}), 5.48-5.55(\mathrm{~m}$, $8 \mathrm{H}), 4.43-4.55(\mathrm{~m}, 8 \mathrm{H}), 2.75-2.82(\mathrm{~m}, 4 \mathrm{H}), 2.37-2.42(\mathrm{~m}, 8 \mathrm{H}), 2.10(\mathrm{~s}, 12 \mathrm{H}), 1.50-1.62(\mathrm{~m}$, $8 \mathrm{H}), 1.23-1.36(\mathrm{~m}, 88 \mathrm{H}), 0.86\left(\mathrm{t},{ }^{3} J_{\mathrm{H}-\mathrm{H}}=8.0 \mathrm{~Hz}, 12 \mathrm{H}\right) \cdot{ }^{13} \mathrm{C}\left\{{ }^{1} \mathrm{H}\right\} \mathrm{NMR}\left(100 \mathrm{MHz}, \mathrm{CD}_{3} \mathrm{CN}\right.$, 
ppm): $\delta=172.81 ; 170.59 ; 156.73 ; 154.38 ; 153.35 ; 151.61 ; 151.56 ; 123.56 ; 122.67 ; 119.48$; $105.33 ; 101.72 ; 97.36 ; 82.18 ; 81.58 ; 31.90 ; 31.63 ; 30.88 ; 29.34 ; 29.24 ; 29.20 ; 29.08 ; 28.95$; $28.64 ; 26.56 ; 22.38 ; 21.55 ; 21.43 ; 21.37 ; 21.24 ; 17.23 ; 13.39 . \mathrm{MS}(\mathrm{ESI}(+))=1507.1[\mathrm{M}-2$ OTf $]^{2+}$. UV-Vis $\left(\mathrm{CH}_{2} \mathrm{Cl}_{2}, 1.0 \times 10^{-5} \mathrm{M}\right): \lambda_{\max }=383 \mathrm{~nm}\left(\varepsilon=0.28 \times 10^{5} \mathrm{M}^{-1} \cdot \mathrm{cm}^{-1}\right) . \mathrm{IR}(\mathrm{KBr})$ $\left(\mathrm{cm}^{-1}\right)=3067(\mathrm{br}, \mathrm{NH}) ; 1698(\mathrm{~s}, \mathrm{NH}) ; 1661(\mathrm{~s}, \mathrm{NH}) ; 1589(\mathrm{~s}, \mathrm{NH}) ; 1260(\mathrm{~s}, \mathrm{CN})$.

4b: Yield $=72 \%$ (0.12 g). ${ }^{1} \mathrm{H}$ NMR (400 MHz, $\left.\mathrm{CD}_{3} \mathrm{CN}, \mathrm{ppm}\right): \delta=12.73$ (s, 4H), 11.98 (s, $4 \mathrm{H}), 11.06\left(\mathrm{t},{ }^{3} J_{\mathrm{H}-\mathrm{H}}=6.0 \mathrm{~Hz}, 4 \mathrm{H}\right), 8.04-8.10(\mathrm{~m}, 8 \mathrm{H}), 7.30-7.37(\mathrm{~m}, 8 \mathrm{H}), 5.85-5.89(\mathrm{~m}, 8 \mathrm{H})$, $5.74(\mathrm{~s}, 44 \mathrm{H}), 5.63-5.69(\mathrm{~m}, 8 \mathrm{H}), 5.62(\mathrm{~s}, 4 \mathrm{H}), 4.38\left(\mathrm{~d},{ }^{3} J_{\mathrm{H}-\mathrm{H}}=5.1 \mathrm{~Hz}, 8 \mathrm{H}\right), 2.82\left(\mathrm{sept},{ }^{3} J_{\mathrm{H}-\mathrm{H}}=\right.$ $5.6 \mathrm{~Hz}, 4 \mathrm{H}), 2.44\left(\mathrm{t},{ }^{3} J_{\mathrm{H}-\mathrm{H}}=8.0 \mathrm{~Hz}, 8 \mathrm{H}\right), 2.10(\mathrm{~s}, 12 \mathrm{H}), 1.55-1.62(\mathrm{~m}, 8 \mathrm{H}), 1.28-1.31(\mathrm{~m}$, $88 \mathrm{H}), 0.88\left(\mathrm{t},{ }^{3} J_{\mathrm{H}-\mathrm{H}}=6.0 \mathrm{~Hz}, 12 \mathrm{H}\right) .{ }^{13} \mathrm{C}\left\{{ }^{1} \mathrm{H}\right\} \mathrm{NMR}\left(100 \mathrm{MHz}, \mathrm{CD}_{3} \mathrm{CN}, \mathrm{ppm}\right): \delta=183.53$; $152.04 ; 128.57 ; 123.48 ; 122.43 ; 117.00 ; 83.08 ; 81.53 ; 31.66 ; 31.34 ; 30.79 ; 29.07 ; 28.96$; $28.82 ; 28.69 ; 28.34 ; 26.23 ; 23.05 ; 22.11 ; 21.11 ; 16.99 ; 13.11 . \mathrm{MS}(\mathrm{ESI}(+))=1557.1[\mathrm{M}-2$ OTf $]^{2+}$. UV-Vis $\left(\mathrm{CH}_{2} \mathrm{Cl}_{2}, 2.0 \times 10^{-6} \mathrm{M}\right): \lambda_{\max }=299 \mathrm{~nm}\left(\varepsilon=3.64 \times 10^{5} \mathrm{M}^{-1} \cdot \mathrm{cm}^{-1}\right), 496 \mathrm{~nm}(\varepsilon=$ $\left.3.39 \times 10^{5} \mathrm{M}^{-1} \cdot \mathrm{cm}^{-1}\right)$. IR (KBr) $\left(\mathrm{cm}^{-1}\right)=3330(\mathrm{br}, \mathrm{NH}) ; 1700(\mathrm{~s}, \mathrm{NH}) ; 1659(\mathrm{~s}, \mathrm{NH}) ; 1521(\mathrm{~s}$, $\mathrm{NH}) ; 1257(\mathrm{~s}, \mathrm{CN})$.

4c: Yield $=72 \%(0.12 \mathrm{~g}) .{ }^{1} \mathrm{H}$ NMR $\left(400 \mathrm{MHz}, \mathrm{CD}_{3} \mathrm{OD}, \mathrm{ppm}\right): \delta=8.24\left(\mathrm{~d},{ }^{3} J_{\mathrm{H}-\mathrm{H}}=5.8 \mathrm{~Hz}\right.$, $8 \mathrm{H}), 7.44\left(\mathrm{~d},{ }^{3} J_{\mathrm{H}-\mathrm{H}}=6.0 \mathrm{~Hz}, 8 \mathrm{H}\right), 6.10\left(\mathrm{~d},{ }^{3} J_{\mathrm{H}-\mathrm{H}}=6.1 \mathrm{~Hz}, 8 \mathrm{H}\right), 5.91\left(\mathrm{~d},{ }^{3} J_{\mathrm{H}-\mathrm{H}}=6.4 \mathrm{~Hz}, 12 \mathrm{H}\right)$, $4.55(\mathrm{~s}, 8 \mathrm{H}), 2.90\left(\mathrm{sept},{ }^{3} J_{\mathrm{H}-\mathrm{H}}=5.6 \mathrm{~Hz}, 4 \mathrm{H}\right), 2.48-2.53(\mathrm{~m}, 8 \mathrm{H}), 2.24(\mathrm{~s}, 12 \mathrm{H}), 1.62-1.71(\mathrm{~m}$, $8 \mathrm{H}), 1.28-1.38(\mathrm{~m}, 88 \mathrm{H}), 0.87-0.90(\mathrm{~m}, 12 \mathrm{H}) .{ }^{13} \mathrm{C}\left\{{ }^{1} \mathrm{H}\right\} \mathrm{NMR}\left(100 \mathrm{MHz}, \mathrm{CD}_{3} \mathrm{OD}, \mathrm{ppm}\right): \delta=$ $152.22 ; 145.84 ; 135.10 ; 128.84 ; 126.16 ; 122.66 ; 119.47 ; 117.30 ; 84.94 ; 83.21 ; 82.76 ; 79.88$; $33.39 ; 31.65 ; 31.20 ; 29.34 ; 29.11 ; 23.33 ; 22.41 ; 21.52 ; 19.92 ; 13.43 ; 13.38$. MS $(\operatorname{ESI}(+))=$ $1625.0[\mathrm{M}-2 \mathrm{OTf}]^{2+}$. UV-Vis $\left(\mathrm{CH}_{2} \mathrm{Cl}_{2}, 2.0 \times 10^{-6} \mathrm{M}\right): \lambda_{\max }=324 \mathrm{~nm}\left(\varepsilon=2.27 \times 10^{5} \mathrm{M}^{-1} \cdot \mathrm{cm}^{-}\right.$ $\left.{ }^{1}\right), 508 \mathrm{~nm}\left(\varepsilon=2.27 \times 10^{5} \mathrm{M}^{-1} \cdot \mathrm{cm}^{-1}\right)$. IR $(\mathrm{KBr})\left(\mathrm{cm}^{-1}\right)=3430(\mathrm{br}, \mathrm{NH}) ; 1707(\mathrm{~s}, \mathrm{NH}) ; 1658(\mathrm{~s}$, $\mathrm{NH}) ; 1498$ (s, NH); 1255 (s, CN).

4d: Yield $=23 \%(0.10 \mathrm{~g}) .{ }^{1} \mathrm{H}$ NMR $\left(400 \mathrm{MHz}, \mathrm{CD}_{3} \mathrm{OD}, \mathrm{ppm}\right): \delta=8.34\left(\mathrm{~d},{ }^{3} J_{\mathrm{H}-\mathrm{H}}=5.8 \mathrm{~Hz}\right.$, $8 \mathrm{H}), 7.32\left(\mathrm{~d},{ }^{3} J_{\mathrm{H}-\mathrm{H}}=6.1 \mathrm{~Hz}, 8 \mathrm{H}\right), 7.17(\mathrm{~s}, 8 \mathrm{H}), 5.90(\mathrm{~s}, 4 \mathrm{H}), 5.80\left(\mathrm{~d},{ }^{3} J_{\mathrm{H}-\mathrm{H}}=6.1 \mathrm{~Hz}, 8 \mathrm{H}\right), 5.57$ $\left(\mathrm{d},{ }^{3} J_{\mathrm{H}-\mathrm{H}}=6.1 \mathrm{~Hz}, 8 \mathrm{H}\right), 4.54(\mathrm{~s}, 8 \mathrm{H}), 2.79\left(\mathrm{sept},{ }^{3} J_{\mathrm{H}-\mathrm{H}}=4.8 \mathrm{~Hz}, 4 \mathrm{H}\right), 2.57$ (quint, ${ }^{3} J_{\mathrm{H}-\mathrm{H}}=6.9$ $\mathrm{Hz}, 8 \mathrm{H}), 2.07(\mathrm{~s}, 12 \mathrm{H}), 1.64-1.71(\mathrm{~m}, 8 \mathrm{H}), 1.26-1.31(\mathrm{~m}, 88 \mathrm{H}), 0.87\left(\mathrm{t},{ }^{3} J_{\mathrm{H}-\mathrm{H}}=6.0 \mathrm{~Hz}, 12 \mathrm{H}\right)$. ${ }^{13} \mathrm{C}\left\{{ }^{1} \mathrm{H}\right\}$ NMR $\left(100 \mathrm{MHz}, \mathrm{CD}_{3} \mathrm{CN}, \mathrm{ppm}\right): \delta=172.67 ; 170.71 ; 156.85 ; 154.31 ; 153.34$; 151.64; 137.30; 125.87; 123.68; 122.68; 119.49; 111.38; 105.27; 103.32; 98.97; 83.88; 82.91; $41.42 ; 31.81 ; 31.65 ; 30.37 ; 29.29 ; 29.12 ; 28.83 ; 28.47 ; 26.36 ; 22.39 ; 21.27 ; 16.28 ; 13.40 . \mathrm{MS}$ 
$(\operatorname{ESI}(+))=1607.0[\mathrm{M}-2 \mathrm{OTf}]^{2+}$. UV-Vis $\left(\mathrm{CH}_{2} \mathrm{Cl}_{2}, 3.6 \times 10^{-6} \mathrm{M}\right): \lambda_{\max }=306 \mathrm{~nm}(\varepsilon=2.38 \mathrm{x}$ $\left.10^{5} \mathrm{M}^{-1} \cdot \mathrm{cm}^{-1}\right), 434 \mathrm{~nm}\left(\varepsilon=1.66 \times 10^{5} \mathrm{M}^{-1} \cdot \mathrm{cm}^{-1}\right), 639 \mathrm{~nm}\left(\varepsilon=0.55 \times 10^{5} \mathrm{M}^{-1} \cdot \mathrm{cm}^{-1}\right), 693 \mathrm{~nm}(\varepsilon$ $\left.=0.62 \times 10^{5} \mathrm{M}^{-1} \cdot \mathrm{cm}^{-1}\right) . \mathrm{IR}(\mathrm{KBr})\left(\mathrm{cm}^{-1}\right)=3334(\mathrm{br}, \mathrm{NH}) ; 1704(\mathrm{~s}, \mathrm{NH}) ; 1659(\mathrm{~s}, \mathrm{NH}) ; 1535$ $(\mathrm{s}, \mathrm{NH}) ; 1274(\mathrm{~s}, \mathrm{CN})$.

5a: Yield $=21 \%(0.09 \mathrm{~g}) .{ }^{1} \mathrm{H}$ NMR $\left(400 \mathrm{MHz}, \mathrm{CD}_{3} \mathrm{OD}, \mathrm{ppm}\right): \delta=8.47\left(\mathrm{~d},{ }^{3} J_{\mathrm{H}-\mathrm{H}}=6.1 \mathrm{~Hz}\right.$, $8 \mathrm{H}), 7.55\left(\mathrm{~d},{ }^{3} J_{\mathrm{H}-\mathrm{H}}=6.0 \mathrm{~Hz}, 8 \mathrm{H}\right), 5.80-5.82(\mathrm{~m}, 12 \mathrm{H}), 5.57\left(\mathrm{~d},{ }^{3} J_{\mathrm{H}-\mathrm{H}}=6.0 \mathrm{~Hz}, 8 \mathrm{H}\right), 2.86$ (sept, $\left.{ }^{3} J_{\mathrm{H}-\mathrm{H}}=6.4 \mathrm{~Hz}, 12 \mathrm{H}\right), 2.52-2.61(\mathrm{~m}, 8 \mathrm{H}), 2.29(\mathrm{~s}, 12 \mathrm{H}), 1.52-1.63(\mathrm{~m}, 20 \mathrm{H}), 1.28-1.40(\mathrm{~m}$, $88 \mathrm{H}), 0.88-0.91(\mathrm{~m}, 12 \mathrm{H}) .{ }^{13} \mathrm{C}\left\{{ }^{1} \mathrm{H}\right\} \mathrm{NMR}\left(100 \mathrm{MHz}, \mathrm{CD}_{3} \mathrm{OD}, \mathrm{ppm}\right): \delta=154.25 ; 143.13$; $125.92 ; 124.68 ; 123.38 ; 120.21 ; 103.59 ; 98.79 ; 83.79 ; 82.16 ; 81.18 ; 78.95 ; 33.07 ; 32.34$; $30.73 ; 30.58 ; 30.48 ; 30.35 ; 30.04 ; 28.55 ; 23.74 ; 22.67 ; 22.65 ; 17.94 ; 14.46 . \mathrm{MS}(\mathrm{ESI}(+))=$ $1535.1[\mathrm{M}-2 \mathrm{OTf}]^{2+}$. UV-Vis $\left(\mathrm{CH}_{2} \mathrm{Cl}_{2}, 1.0 \times 10^{-5} \mathrm{M}\right): \lambda_{\max }=387 \mathrm{~nm}\left(\varepsilon=0.28 \times 10^{5} \mathrm{M}^{-1} \cdot \mathrm{cm}^{-}\right.$ $\left.{ }^{1}\right)$. IR $(\mathrm{KBr})\left(\mathrm{cm}^{-1}\right)=3276(\mathrm{br}, \mathrm{NH}) ; 1660(\mathrm{~s}, \mathrm{NH}) ; 1633(\mathrm{~s}, \mathrm{NH}) ; 1242(\mathrm{~s}, \mathrm{CN})$.

5b: Yield $=61 \%(0.12 \mathrm{~g}) .{ }^{1} \mathrm{H}$ NMR $\left(400 \mathrm{MHz}, \mathrm{CD}_{3} \mathrm{OD}, \mathrm{ppm}\right): \delta=8.21-8.26(\mathrm{~m}, 8 \mathrm{H}), 7.44-$ $7.49(\mathrm{~m}, 8 \mathrm{H}), 5.93-6.00(\mathrm{~m}, 12 \mathrm{H}), 5.74-5.79(\mathrm{~m}, 12 \mathrm{H}), 2.86\left(\mathrm{sept},{ }^{3} J_{\mathrm{H}-\mathrm{H}}=7.7 \mathrm{~Hz}, 4 \mathrm{H}\right), 2.47-$ $2.53(\mathrm{~m}, 4 \mathrm{H}), 2.17(\mathrm{~s}, 12 \mathrm{H}), 1.52-1.66(\mathrm{~m}, 20 \mathrm{H}), 1.29-1.35(\mathrm{~m}, 88 \mathrm{H}), 0.87-0.91(\mathrm{~m}, 12 \mathrm{H})$. ${ }^{13} \mathrm{C}\left\{{ }^{1} \mathrm{H}\right\}$ NMR $\left(100 \mathrm{MHz}, \mathrm{CD}_{3} \mathrm{OD}, \mathrm{ppm}\right): \delta=185.48 ; 185.37 ; 154.15 ; 153.91 ; 153.85 ;$ $126.52 ; 125.02 ; 123.35 ; 120.18 ; 117.01 ; 105.06 ; 105.01 ; 104.78 ; 102.66 ; 99.87 ; 99.67 ; 84.76$; $84.69 ; 83.58 ; 83.31 ; 83.18 ; 33.10 ; 33.07 ; 32.55 ; 30.79 ; 30.75 ; 30.66 ; 30.52 ; 30.49 ; 30.42$; $30.11 ; 28.49 ; 23.77 ; 22.56 ; 22.51 ; 21.72 ; 18.20 ; 18.15 ; 14.49 . \mathrm{MS}(\mathrm{ESI}(+))=1585.1[\mathrm{M}-2$ $\mathrm{OTf}^{2+}$. UV-Vis $\left(\mathrm{CH}_{2} \mathrm{Cl}_{2}, 2.2 \times 10^{-6} \mathrm{M}\right): \lambda_{\max }=298 \mathrm{~nm}\left(\varepsilon=3.42 \times 10^{5} \mathrm{M}^{-1} \cdot \mathrm{cm}^{-1}\right), 496 \mathrm{~nm}(\varepsilon=$ $\left.3.07 \times 10^{5} \mathrm{M}^{-1} \cdot \mathrm{cm}^{-1}\right)$. IR (KBr) $\left(\mathrm{cm}^{-1}\right)=3293(\mathrm{br}, \mathrm{NH}) ; 1700(\mathrm{~s}, \mathrm{NH}) ; 1658(\mathrm{~s}, \mathrm{NH}) ; 1523(\mathrm{~s}$, $\mathrm{NH}) ; 1257$ (s, CN).

5c: Yield $=57 \%(0.12 \mathrm{~g}) .{ }^{1} \mathrm{H}$ NMR (400 MHz, $\left.\mathrm{CD}_{3} \mathrm{OD}, \mathrm{ppm}\right): \delta=8.10-8.16(\mathrm{~m}, 8 \mathrm{H}), 7.38$ $7.40(\mathrm{~m}, 8 \mathrm{H}), 5.89-5.92(\mathrm{~m}, 12 \mathrm{H}), 5.73-5.77(\mathrm{~m}, 8 \mathrm{H}), 3.47-3.50(\mathrm{~m}, 4 \mathrm{H}), 2.86\left(\mathrm{sept},{ }^{3} J_{\mathrm{H}-\mathrm{H}}=\right.$ $6.0 \mathrm{~Hz}, 4 \mathrm{H}), 2.47-2.53(\mathrm{~m}, 16 \mathrm{H}), 2.24(\mathrm{~s}, 12 \mathrm{H}), 1.24-1.56(\mathrm{~m}, 96 \mathrm{H}), 0.87-0.91(\mathrm{~m}, 12 \mathrm{H})$. ${ }^{13} \mathrm{C}\left\{{ }^{1} \mathrm{H}\right\}$ NMR $\left(100 \mathrm{MHz}, \mathrm{CD}_{3} \mathrm{OD}, \mathrm{ppm}\right): \delta=179.07 ; 153.80 ; 125.84 ; 125.34 ; 123.34$; $120.17 ; 105.42 ; 84.74 ; 83.97 ; 33.11 ; 32.71 ; 30.74 ; 30.55 ; 30.02 ; 28.50 ; 23.76 ; 22.52 ; 21.64$; 21.39; 18.20; 14.49. MS $(\operatorname{ESI}(+))=1652.9\left[\mathrm{M}-2 \mathrm{OTf}^{2+}\right.$. UV-Vis $\left(\mathrm{CH}_{2} \mathrm{Cl}_{2}, 2.3 \times 10^{-6} \mathrm{M}\right)$ : $\lambda_{\max }=325 \mathrm{~nm}\left(\varepsilon=2.31 \times 10^{5} \mathrm{M}^{-1} \cdot \mathrm{cm}^{-1}\right), 506 \mathrm{~nm}\left(\varepsilon=2.40 \times 10^{5} \mathrm{M}^{-1} \cdot \mathrm{cm}^{-1}\right)$. IR $(\mathrm{KBr})\left(\mathrm{cm}^{-1}\right)=$ 3336 (br, NH); 1704 (s, NH); 1658 (s, NH); 1497 (s, NH); 1256 (s, CN). 
5d: Yield $=21 \%(0.09 \mathrm{~g}) .{ }^{1} \mathrm{H}$ NMR $\left(400 \mathrm{MHz}, \mathrm{CD}_{3} \mathrm{OD}, \mathrm{ppm}\right): \delta=8.38(\mathrm{~s}, 8 \mathrm{H}), 7.40-7.44(\mathrm{~m}$, $8 \mathrm{H}), 7.15-7.18(\mathrm{~m}, 8 \mathrm{H}), 5.81-5.89(\mathrm{~m}, 12 \mathrm{H}), 5.56-5.59(\mathrm{~m}, 12 \mathrm{H}), 2.79\left(\mathrm{sept},{ }^{3} J_{\mathrm{H}-\mathrm{H}}=7.2 \mathrm{~Hz}\right.$, $4 \mathrm{H}), 2.46-2.52(\mathrm{~m}, 8 \mathrm{H}), 2.07(\mathrm{~s}, 12 \mathrm{H}), 1.46-1.63(\mathrm{~m}, 20 \mathrm{H}), 1.26-1.36(\mathrm{~m}, 88 \mathrm{H}), 0.86-0.91(\mathrm{~m}$, 12H). ${ }^{13} \mathrm{C}\left\{{ }^{1} \mathrm{H}\right\}$ NMR $\left(100 \mathrm{MHz}, \mathrm{CD}_{3} \mathrm{OD}, \mathrm{ppm}\right): \delta=153.00 ; 138.56 ; 123.42 ; 123.03 ; 120.26$; $104.86 ; 100.85 ; 85.73 ; 83.95 ; 33.12 ; 33.08 ; 31.98 ; 30.73 ; 30.59 ; 30.49 ; 30.06 ; 23.80 ; 22.45$; 17.35; 14.52. MS $(\mathrm{ESI}(+))=1635.1[\mathrm{M}-2 \mathrm{OTf}]^{2+}$. UV-Vis $\left(\mathrm{CH}_{2} \mathrm{Cl}_{2}, 3.1 \times 10^{-6} \mathrm{M}\right): \lambda_{\max }=$ $434 \mathrm{~nm}\left(\varepsilon=1.57 \times 10^{5} \mathrm{M}^{-1} \cdot \mathrm{cm}^{-1}\right), 641 \mathrm{~nm}\left(\varepsilon=0.51 \times 10^{5} \mathrm{M}^{-1} \cdot \mathrm{cm}^{-1}\right), 693 \mathrm{~nm}\left(\varepsilon=0.51 \times 10^{5}\right.$ $\left.\mathrm{M}^{-1} \cdot \mathrm{cm}^{-1}\right)$. IR $(\mathrm{KBr})\left(\mathrm{cm}^{-1}\right)=3286(\mathrm{br}, \mathrm{NH}) ; 1703(\mathrm{~s}, \mathrm{NH}) ; 1659(\mathrm{~s}, \mathrm{NH}) ; 1532(\mathrm{~s}, \mathrm{NH}) ; 1275$ $(\mathrm{s}, \mathrm{CN})$.

6a: Yield $=23 \%(0.09 \mathrm{~g}) .{ }^{1} \mathrm{H}$ NMR $\left(400 \mathrm{MHz}, \mathrm{CD}_{3} \mathrm{OD}, \mathrm{ppm}\right): \delta=8.41\left(\mathrm{~d},{ }^{3} J_{\mathrm{H}-\mathrm{H}}=5.8 \mathrm{~Hz}\right.$, $8 \mathrm{H}), 7.50\left(\mathrm{~d},{ }^{3} J_{\mathrm{H}-\mathrm{H}}=5.8 \mathrm{~Hz}, 8 \mathrm{H}\right), 6.10(\mathrm{~s}, 4 \mathrm{H}), 5.80\left(\mathrm{~d},{ }^{3} J_{\mathrm{H}-\mathrm{H}}=5.9 \mathrm{~Hz}, 8 \mathrm{H}\right), 5.58\left(\mathrm{~d},{ }^{3} J_{\mathrm{H}-\mathrm{H}}=\right.$ $5.9 \mathrm{~Hz}, 8 \mathrm{H}), 3.58\left(\mathrm{t},{ }^{3} J_{\mathrm{H}-\mathrm{H}}=6.6 \mathrm{~Hz}, 8 \mathrm{H}\right), 3.00\left(\mathrm{t},{ }^{3} J_{\mathrm{H}-\mathrm{H}}=6.7 \mathrm{~Hz}, 8 \mathrm{H}\right), 2.84\left(\mathrm{sept},{ }^{3} J_{\mathrm{H}-\mathrm{H}}=6.9\right.$ $\mathrm{Hz}, 4 \mathrm{H}), 2.60\left(\mathrm{t},{ }^{3} J_{\mathrm{H}-\mathrm{H}}=7.7 \mathrm{~Hz}, 8 \mathrm{H}\right), 2.13(\mathrm{~s}, 12 \mathrm{H}), 1.64-1.68(\mathrm{~m}, 8 \mathrm{H}), 1.28-1.40(\mathrm{~m}, 88 \mathrm{H})$, $0.90\left(\mathrm{t},{ }^{3} J_{\mathrm{H}-\mathrm{H}}=6.9 \mathrm{~Hz}, 12 \mathrm{H}\right) .{ }^{13} \mathrm{C}\left\{{ }^{1} \mathrm{H}\right\} \mathrm{NMR}\left(100 \mathrm{MHz}, \mathrm{CDCl}_{3}, \mathrm{ppm}\right): \delta=166.16 ; 152.41$; $127.65 ; 126.40 ; 118.76 ; 102.01 ; 97.31 ; 82.36 ; 80.46 ; 79.94 ; 77.48 ; 47.12 ; 46.90 ; 31.62$; $29.28 ; 29.22 ; 29.14 ; 29.02 ; 28.93 ; 22.29 ; 21.19 ; 21.15 ; 16.50 ; 12.99 . \operatorname{MS}(\operatorname{ESI}(+))=1535.1$ $[\mathrm{M}-2 \mathrm{OTf}]^{2+}$. UV-Vis $\left(\mathrm{CH}_{2} \mathrm{Cl}_{2}, 1.0 \times 10^{-5} \mathrm{M}\right): \lambda_{\max }=383 \mathrm{~nm}\left(\varepsilon=0.26 \times 10^{5} \mathrm{M}^{-1} \cdot \mathrm{cm}^{-1}\right) . \mathrm{IR}$ $(\mathrm{KBr})\left(\mathrm{cm}^{-1}\right)=3338(\mathrm{br}, \mathrm{NH}) ; 1706(\mathrm{~s}, \mathrm{NH}) ; 1657(\mathrm{~s}, \mathrm{NH}) ; 1632(\mathrm{~s}, \mathrm{NH}) ; 1258(\mathrm{~s}, \mathrm{CN})$.

6b: Yield $=65 \%(0.13 \mathrm{~g}) .{ }^{1} \mathrm{H}$ NMR $\left(400 \mathrm{MHz}, \mathrm{CD}_{3} \mathrm{OD}, \mathrm{ppm}\right): \delta=8.17\left(\mathrm{~d},{ }^{3} J_{\mathrm{H}-\mathrm{H}}=5.8 \mathrm{~Hz}\right.$, $8 \mathrm{H}), 7.38\left(\mathrm{~d},{ }^{3} J_{\mathrm{H}-\mathrm{H}}=5.7 \mathrm{~Hz}, 8 \mathrm{H}\right), 6.01\left(\mathrm{~d},{ }^{3} J_{\mathrm{H}-\mathrm{H}}=6.0 \mathrm{~Hz}, 4 \mathrm{H}\right), 5.79\left(\mathrm{~d},{ }^{3} J_{\mathrm{H}-\mathrm{H}}=6.1 \mathrm{~Hz}, 12 \mathrm{H}\right)$, 3.13-3.23 (m, 8H), 2.83-2.90 (m, 4H), $2.57\left(\mathrm{t},{ }^{3} J_{\mathrm{H}-\mathrm{H}}=7.6 \mathrm{~Hz}, 8 \mathrm{H}\right), 2.17(\mathrm{~s}, 6 \mathrm{H}), 1.66-1.72(\mathrm{~m}$, $8 \mathrm{H}), 1.28-1.36(\mathrm{~m}, 96 \mathrm{H}), 0.90\left(\mathrm{t},{ }^{3} J_{\mathrm{H}-\mathrm{H}}=6.5 \mathrm{~Hz}, 12 \mathrm{H}\right) .{ }^{13} \mathrm{C}\left\{{ }^{1} \mathrm{H}\right\} \mathrm{NMR}\left(100 \mathrm{MHz}, \mathrm{CD}_{3} \mathrm{OD}\right.$, ppm): $\delta=153.78 ; 127.89 ; 123.44 ; 120.27 ; 84.79 ; 83.13 ; 33.11 ; 32.60 ; 30.79 ; 30.54 ; 23.78$; 22.54; 14.49. MS $(\operatorname{ESI}(+))=1585.1[\mathrm{M}-2 \mathrm{OTf}]^{2+}$. UV-Vis $\left(\mathrm{CH}_{2} \mathrm{Cl}_{2}, 2.2 \times 10^{-6} \mathrm{M}\right): \lambda_{\max }=$ $497 \mathrm{~nm}\left(\varepsilon=2.23 \times 10^{5} \mathrm{M}^{-1} \cdot \mathrm{cm}^{-1}\right)$. IR $(\mathrm{KBr})\left(\mathrm{cm}^{-1}\right)=3421(\mathrm{br}, \mathrm{NH}) ; 1524(\mathrm{~s}, \mathrm{NH}) ; 1257(\mathrm{~s}$, $\mathrm{CN})$.

6c: Yield $=53 \%(0.11 \mathrm{~g}) .{ }^{1} \mathrm{H}$ NMR (400 MHz, $\left.\mathrm{CD}_{3} \mathrm{CN}, \mathrm{ppm}\right): \delta=11.74(\mathrm{~s}, 4 \mathrm{H}), 10.75$ (s, $4 \mathrm{H}), 8.12\left(\mathrm{~d},{ }^{3} J_{\mathrm{H}-\mathrm{H}}=5.9 \mathrm{~Hz}, 8 \mathrm{H}\right), 7.30\left(\mathrm{~d},{ }^{3} J_{\mathrm{H}-\mathrm{H}}=5.8 \mathrm{~Hz}, 8 \mathrm{H}\right), 5.96\left(\mathrm{~d},{ }^{3} J_{\mathrm{H}-\mathrm{H}}=6.3 \mathrm{~Hz}, 8 \mathrm{H}\right)$, $5.76\left(\mathrm{~d},{ }^{3} J_{\mathrm{H}-\mathrm{H}}=6.1 \mathrm{~Hz}, 8 \mathrm{H}\right), 5.58(\mathrm{~s}, 4 \mathrm{H}), 3.06-3.15(\mathrm{~m}, 8 \mathrm{H}), 2.87\left(\mathrm{sept},{ }^{3} J_{\mathrm{H}-\mathrm{H}}=6.9 \mathrm{~Hz}, 12 \mathrm{H}\right)$, $2.50\left(\mathrm{t},{ }^{3} J_{\mathrm{H}-\mathrm{H}}=7.6 \mathrm{~Hz}, 8 \mathrm{H}\right), 2.10(\mathrm{~s}, 12 \mathrm{H}) ; 1.64-1.66(\mathrm{~m}, 8 \mathrm{H}) ; 1.29-1.38(\mathrm{~m}, 88 \mathrm{H}), 0.90\left(\mathrm{t},{ }^{3} J_{\mathrm{H}-}\right.$ $\mathrm{H}=6.5 \mathrm{~Hz}, 12 \mathrm{H}) .{ }^{13} \mathrm{C}\left\{{ }^{1} \mathrm{H}\right\} \mathrm{NMR}\left(100 \mathrm{MHz}, \mathrm{CD}_{3} \mathrm{CN}, \mathrm{ppm}\right): \delta=152.23 ; 128.57 ; 126.27$; 
$122.43 ; 119.24 ; 83.09 ; 82.04 ; 31.36 ; 30.94 ; 29.07 ; 28.81 ; 28.76 ; 28.20 ; 26.13 ; 22.11 ; 21.05$; 13.12. MS $(\operatorname{ESI}(+))=1653.0[\mathrm{M}-2 \mathrm{OTf}]^{2+}$. UV-Vis $\left(\mathrm{CH}_{2} \mathrm{Cl}_{2}, 2.1 \times 10^{-5} \mathrm{M}\right): \lambda_{\max }=326 \mathrm{~nm}$ $\left(\varepsilon=2.20 \times 10^{5} \mathrm{M}^{-1} \cdot \mathrm{cm}^{-1}\right), 509 \mathrm{~nm}\left(\varepsilon=2.25 \times 10^{5} \mathrm{M}^{-1} \cdot \mathrm{cm}^{-1}\right)$. IR $(\mathrm{KBr})\left(\mathrm{cm}^{-1}\right)=3367(\mathrm{br}, \mathrm{NH})$; 1703 (s, NH); 1658 (s, NH); 1498 (s, NH); 1225 (s, CN).

6d: Yield $=46 \%(0.10 \mathrm{~g}) .{ }^{1} \mathrm{H}$ NMR $\left(400 \mathrm{MHz}, \mathrm{CD}_{2} \mathrm{Cl}_{2}, \mathrm{ppm}\right): \delta=8.18\left(\mathrm{~d},{ }^{3} J_{\mathrm{H}-\mathrm{H}}=5.6 \mathrm{~Hz}\right.$, $8 \mathrm{H}), 7.23-7.25(\mathrm{~m}, 8 \mathrm{H}), 7.15(\mathrm{~s}, 8 \mathrm{H}), 5.60\left(\mathrm{~d},{ }^{3} J_{\mathrm{H}-\mathrm{H}}=6.1 \mathrm{~Hz}, 12 \mathrm{H}\right), 5.42\left(\mathrm{~d},{ }^{3} J_{\mathrm{H}-\mathrm{H}}=6.0 \mathrm{~Hz}\right.$, $12 \mathrm{H}), 3.30\left(\mathrm{q},{ }^{3} J_{\mathrm{H}-\mathrm{H}}=6.6 \mathrm{~Hz}, 4 \mathrm{H}\right), 2.80-2.87(\mathrm{~m}, 4 \mathrm{H}), 2.77\left(\mathrm{q},{ }^{3} J_{\mathrm{H}-\mathrm{H}}=6.7 \mathrm{~Hz}, 4 \mathrm{H}\right), 2.47-2.54$ $(\mathrm{m}, 4 \mathrm{H}), 2.07(\mathrm{~s}, 12 \mathrm{H}), 1.27-1.34(\mathrm{~m}, 88 \mathrm{H}), 0.89\left(\mathrm{t},{ }^{3} J_{\mathrm{H}-\mathrm{H}}=6.5 \mathrm{~Hz}, 12 \mathrm{H}\right) .{ }^{13} \mathrm{C}\left\{{ }^{1} \mathrm{H}\right\} \mathrm{NMR}(100$ $\left.\mathrm{MHz}, \mathrm{CD}_{2} \mathrm{Cl}_{2}, \mathrm{ppm}\right): \delta=172.56 ; 171.51 ; 170.62 ; 156.24 ; 155.86 ; 154.50 ; 153.76 ; 153.00 ;$ $152.79 ; 152.21 ; 151.83 ; 151.65 ; 137.22 ; 136.83 ; 126.24 ; 126.05 ; 125.89 ; 122.70 ; 119.51$; $112.23 ; 111.62 ; 105.18 ; 103.20 ; 103.03 ; 99.11 ; 98.31 ; 84.06 ; 83.70 ; 83.05 ; 82.76 ; 39.95$; $34.45 ; 31.94 ; 31.71 ; 31.63 ; 30.40 ; 30.36 ; 29.42 ; 29.33 ; 29.23 ; 29.18 ; 29.15 ; 29.07 ; 29.01$; $28.72 ; 28.46 ; 26.39 ; 22.45 ; 22.39 ; 21.29 ; 21.11 ; 16.30 . \mathrm{MS}(\mathrm{ESI}(+))=1635.1[\mathrm{M}-2 \text { OTf }]^{2+}$. UV-Vis $\left(\mathrm{CH}_{2} \mathrm{Cl}_{2}, 2.8 \times 10^{-6} \mathrm{M}\right): \lambda_{\max }=310 \mathrm{~nm}\left(\varepsilon=2.56 \times 10^{5} \mathrm{M}^{-1} \cdot \mathrm{cm}^{-1}\right), 435 \mathrm{~nm}(\varepsilon=1.83 \mathrm{x}$ $\left.10^{5} \mathrm{M}^{-1} \cdot \mathrm{cm}^{-1}\right), 644 \mathrm{~nm}\left(\varepsilon=0.55 \times 10^{5} \mathrm{M}^{-1} \cdot \mathrm{cm}^{-1}\right), 696 \mathrm{~nm}\left(\varepsilon=0.58 \times 10^{5} \mathrm{M}^{-1} \cdot \mathrm{cm}^{-1}\right)$. IR $(\mathrm{KBr})$ $\left(\mathrm{cm}^{-1}\right)=3336(\mathrm{br}, \mathrm{NH}) ; 1710(\mathrm{~s}, \mathrm{NH}) ; 1659(\mathrm{~s}, \mathrm{NH}) ; 1535(\mathrm{~s}, \mathrm{NH}) ; 1274(\mathrm{~s}, \mathrm{CN})$.

\section{Acknowledgements}

RD thanks the Swiss National Science Foundation (Grant 200020-152716) for financial support.

\section{References}

[1] (a) T.A. Evans, K.R. Seddon, Chem. Commun. (1997) 2023-2024;

(b) E.A. Archer, H. Gong, M.J. Krische, Tetrahedron 57 (2001) 1139-1159;

(c) J.L. Sessler, C.M. Lawrence, J. Jayawickramarajah, Chem. Soc. Rev. 36 (2007) 314-325;

(d) H.-J. Schneider, Angew. Chem. Int. Ed. 48 (2009) 3924-3977.

[2] (a) M.J. Krische, J.-M. Lehn, Struct. Bonding 96 (2000) 3-29;

(b) P.S. Corbin, L.J. Lawless, Z. Li, Y. Ma, M.J. Witmer, S.C. Zimmerman, Proc. Natl. Acad. Sci. USA, 99 (2002) 5099-5104;

(c) J.L. Sessler, J. Jayawickramarajah, Chem. Commun. (2005) 1939-1949.

[3] (a) H. Zeng, R.S. Miller, R.A. Flowers, B. Gong, J. Am. Chem. Soc. 122 (2000) 2635-2644;

(b) T. Moriuchi, T. Tamura, T. Hirao, J. Am. Chem. Soc. 124 (2002) 9356-9357;

(c) H. Zeng, X. Yang, A.L. Brown, S. Martinovic, R.D. Smith, B. Gong, Chem. Commun. (2003) 1556-1557; 
(d) B. Gong, Acc. Chem. Res. 45 (2012) 2077-2087;

(e) N. Roy, E. Buhler, J.-M. Lehn, Chem. Eur. J. 19 (2013) 8814-8820;

(f) H. Zeng, Q. Huang, J. Liu, Y. Huang, J. Zhou, S. Zhao, Z. Lu, Chin. J. Chem. 34 (2016) 387-396.

[4] (a) Y. Ma, S.V. Kolotuchin, S.C. Zimmerman, J. Am. Chem. Soc. 124 (2002) 13757-13769;

(b) X.-B. Shao, X.-K. Jiang, S.-Z. Zhu, Z.-T. Li, Tetrahedron 60 (2004) 9155-9162;

(c) S. Djurdjevic, D.A. Leigh, H. McNab, S. Parsons, G. Teobaldi, F. Zerbetto, J. Am. Chem. Soc. 129 (2007) 476-477;

(d) H.-B. Wang, B.P. Mudraboyina, J.A. Wisner, Chem. Eur. J. 18 (2012) 1322-1327;

(e) Y.-F. Han, W.-Q. Chen, H.-B. Wang, Y.-X. Yuan, N.-N. Wu, X.-Z. Song, L. Yang, Chem. Eur. J. 20 (2014) 16980-16986;

(f) C. Montoro-García, J. Camacho-García, A.M. López-Pérez, N. Bilbao, S. RomeroPérez, M.J. Mayoral, D. González-Rodríguez, Angew. Chem. Int. Ed. 54 (2015) 6780-6784;

(g) M. Papmeyer, C.A. Vuilleumier, G.M. Pavan, K.O. Zhurov, K. Severin, Angew. Chem. Int. Ed. 55 (2016) 1685-1689.

[5] (a) C. Schmuck, W. Wienand, Angew. Chem. Int. Ed. 40 (2001) 4363-4369;

(b) R.P. Sijbesma, E.W. Meijer, Chem. Commun. (2003) 5-16;

(c) Y. Hisamatsu, N. Shirai, S.-i. Ikeda, K. Odashima, Org. Lett. 11 (2009) 4342-4345;

(d) P. Zhang, H. Chu, X. Li, W. Feng, P. Deng, L. Yuan, B. Gong, Org. Lett. 13 (2011) 54-57;

(e) B.A. Blight, C.A. Hunter, D.A. Leigh, A. McNab, P.I.T. Thomson, Nat. Chem. 3 (2011) 244-248;

(f) M.L. Pellizzaro, S.A. Barrett, J. Fisher, A.J. Wilson, Org. Biomol. Chem. 10 (2012) 4899-4906;

(g) D.A. Leigh, C.C. Robertson, A.M.Z. Slawin, P.I.T. Thomson, J. Am. Chem. Soc. 135 (2013) 9939-9943;

(h) P. Otte, J. Taubitz, U. Lüning, Eur. J. Org. Chem. (2013) 2130-2139.

[6] (a) W. L. Jorgensen, J. Pranata, J. Am. Chem. Soc. 112 (1990) 2008-2010;

(b) J. Pranata, S.G. Wierschke, W.L. Jorgensen, J. Am. Chem. Soc. 113 (1991) 2810-2819;

(c) L.J. Prins, D.N. Reinhoudt, P. Timmerman, Angew. Chem. Int. Ed. 40 (2001) 2382-2426;

(d) T. Rehm, C. Schmuck, Chem. Commun. (2008) 801-813.

[7] (a) R.P. Sijbesma, F.H. Beijer, L. Brunsveld, B.J.B. Folmer, J.H.K. Ky Hirschberg, R.F.M. Lange, J.K.L. Lowe, E.W. Meijer, Science, 278 (1997) 1601-1604;

(b) J.J. González, S. González, E.M. Priego, C. Luo, D.M. Guldi, J. de Mendoza, N. Martín, Chem. Commun. (2001) 163-164;

(c) K. Yamauchi, J.R. Lizotte, T.E. Long, Macromolecules 36 (2003) 1083-1088;

(d) J. T. Roland, Z. Guan, J. Am. Chem. Soc. 126 (2004) 14328-14329;

(e) L.L. Crowe, K.M. Solntsev, L.M. Tolbert, Langmuir, 23 (2007) 6227-6232; 
(f) J. Cui, D. Wang, K. Koynov, A. del Campo, ChemPhysChem 14 (2013) 2932-2938;

(g) Y. Lin, G. Li, J. Mat. Chem. B 2 (2014) 6878-6885;

(h) S. Bobade, Y. Wang, J. Mays, D. Baskaran, Macromolecules 47 (2014) 5040-5050.

[8] (a) M.D. Pluth, K.N. Raymond, Chem. Soc. Rev. 36 (2007) 161-171;

(b) M.D. Ward, Chem. Commun. (2009) 4487-4499;

(c) M. Yoshizawa, J.K. Klosterman, M. Fujita, Angew. Chem. Int. Ed. 48 (2009) 3418-3438;

(d) H. Amouri, C. Desmarets, J. Moussa, Chem. Rev. 112 (2012) 2015-2041;

(e) M.M.J. Smulders, I.A. Riddell, C. Browne, J.R. Nitschke, Chem. Soc. Rev. 42 (2013) 1728-1754;

(f) P. Thanasekaran, C.-H. Lee, K.-L. Lu, Coord. Chem. Rev., 280 (2014) 96-175;

(g) T.R. Cook, P.J. Stang, Chem. Rev. 115 (2015) 7001-7045.

[9] (a) K. Severin, Chem. Commun. (2006) 3859-3867;

(b) B. Therrien, Eur. J. Inorg. Chem. (2009) 2445-2453;

(c) A.K. Singh, D.S. Pandey, Q. Xu, P. Braunstein, Coord. Chem. Rev. 270-271 (2014) 31-56;

(d) B. Therrien, Top. Curr. Chem., 319 (2012) 35-55.

[10] B. Kilbas, S. Mirtschin, T. Riis-Johannessen, R. Scopelliti, K. Severin, Inorg. Chem. 51 (2012) 5795-5804.

[11] (a) S. Korn, W. S. Sheldrick, J. Chem. Soc., Dalton Trans. (1997) 2191-2200;

(b) T. Habereder, M. Warchhold, H. Nöth, K. Severin, Angew. Chem. Int. Ed. 38 (1999) 3225-3228;

(c) K. Yamanari, R. Ito, S. Yamamoto, T. Konno, A. Fuyuhiro, K. Fujioka, R. Arakawa, Inorg. Chem. 41 (2002) 6824-6830;

(d) T. Brasey, R. Scopelliti, K. Severin, Chem. Commun. (2006) 3308-3310.

[12] (a) N.P.E. Barry, N.H. Abd Karim, R. Vilar, B. Therrien, Dalton Trans. (2009) 10717-10719;

(b) F. Schmitt, J. Freudenreich, N.P.E. Barry, L. Juillerat-Jeanneret, G. Süss-Fink, B. Therrien, J. Am. Chem. Soc. 134 (2012) 754-757.

[13] C. Schouwey, R. Scopelliti, K. Severin, Chem. Eur. J. 19 (2013) 6274-6281.

[14] S. Mirtschin, A. Slabon-Turski, R. Scopelliti, A.H. Velders, K. Severin, J. Am. Chem. Soc. 132 (2010) 14004-14005.

[15] (a) H. Yan, G. Süss-Fink, A. Neels, H. Stoeckli-Evans, J. Chem. Soc., Dalton Trans. (1997) 4345-4350;

(b) F. Linares, M. A. Galindo, S. Galli, M. Angustias Romero, J.A.R. Navarro, E. Barea, Inorg. Chem. 48 (2009) 7413-7420;

(c) B. Kilbas, S. Mirtschin, R. Scopelliti, K. Severin, Chem. Sci. 3 (2012) 701-704;

(d) A. Pitto-Barry, N.P.E. Barry, V. Russo, B. Heinrich, B. Donnio, B. Therrien, R. Deschenaux, J. Am. Chem. Soc. 136 (2014) 17616-17625;

(e) A. Dubey, A. Mishra, J.W. Min, M.H. Lee, H. Kim, P.J. Stang, K.-W. Chi, Inorg. Chim. Acta 423 (2014) 326-331; 
(f) Y.H. Song, N. Singh, J. Jung, H. Kim, E.-H. Kim, H.-K. Cheong, Y. Kim, K.-W. Chi, Angew. Chem. Int. Ed. 55 (2016) 2007-2011.

[16] (a) B. Therrien, G. Süss-Fink, P. Govindaswamy, A.K. Renfrew, P.J. Dyson, Angew. Chem. Int. Ed. 47 (2008) 3773-3776;

(b) M. Wang, V. Vajpayee, S. Shanmugaraju, Y.-R. Zheng, Z. Zhao, H. Kim, P.S. Mukherjee, K.-W. Chi, P.J. Stang, Inorg. Chem. 50 (2011) 1506-1512;

(c) V. Vajpayee, S.m. Lee, J.W. Park, A. Dubey, H. Kim, T.R. Cook, P.J. Stang, K.W. Chi, Organometallics, 32 (2013) 1563-1566;

(d) A. Garci, A. A. Dobrov, T. Riedel, E. Orhan, P.J. Dyson, V.B. Arion, B. Therrien, Organometallics 33 (2014) 3813-3822.

[17] D. Appavoo, N. Raja, R. Deschenaux, B. Therrien, D. Carnevale, Dalton Trans. 45 (2016) 1410-1421.

[18] L.J. Marshall, J. de Mendoza, Org. Lett. 15 (2013) 1548-1551.

[19] (a) J. Freudenreich, J. Furrer, G. Süss-Fink, B. Therrien, Organometallics, 30 (2011) 942-951;

(b) A. Garci, S. Marti, S. Schürch, B. Therrien, RSC Adv. 4 (2014) 8597-8604;

(c) A. Garci, G. Gupta, C. Dalvit, B. Therrien, Eur. J. Inorg. Chem. (2014) 5651-5661.

[20] J. Mattsson, P. Govindaswamy, J. Furrer, Y. Sei, K. Yamaguchi, G. Süss-Fink, B. Therrien, Organometallics 27 (2008) 4346-4356.

[21] N.P.E. Barry, B. Therrien, Eur. J. Inorg. Chem. (2009) 4695-4700. 


\section{Graphical abstract}

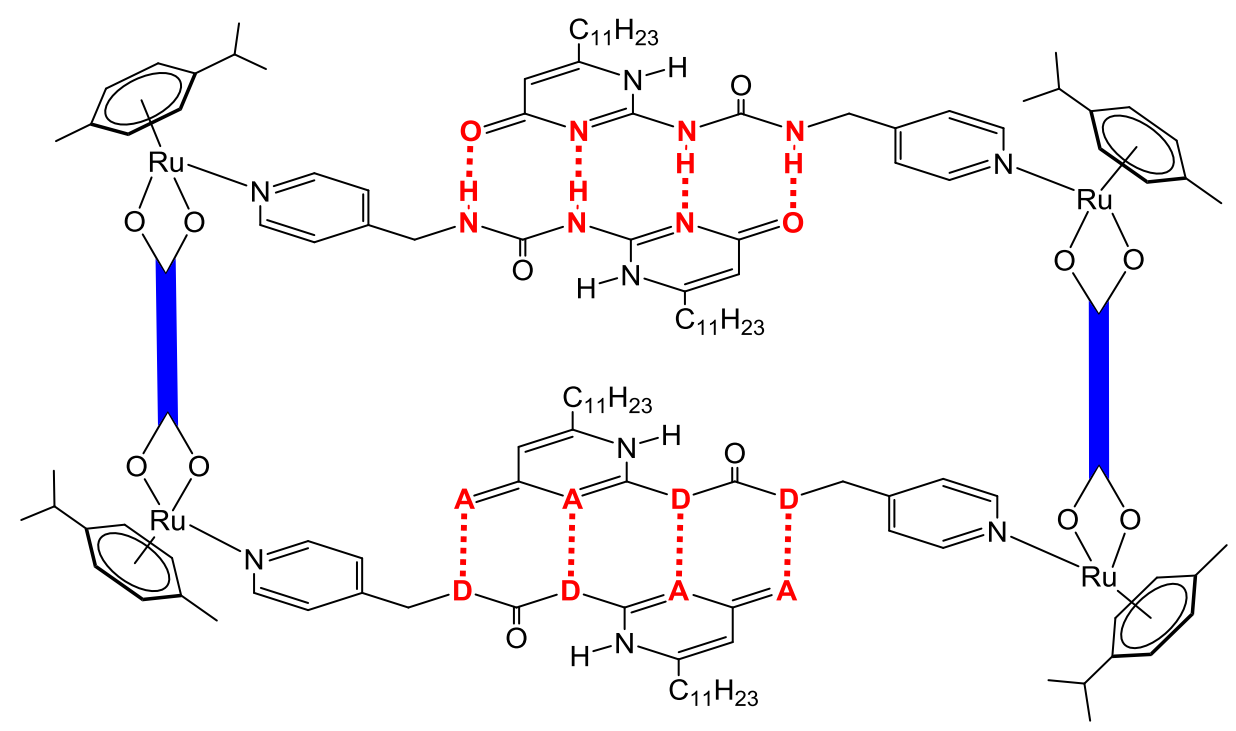

Twelve arene ruthenium metalla-cycles were obtained by combining three ureido-pyrimidone ligands and four dinuclear arene ruthenium complexes. Hydrogen-bonds between the ureidopyrimidone moieties allow the formation of these mixed coordination- and hydrogen-bonded systems. This study confirms the effectiveness of hydrogen-bonds in constructing supramolecular assemblies. 